\title{
ON THE STRUCTURE AND REPRESENTATIONS OF MAX-STABLE PROCESSES
}

\author{
YIZAO WANG ${ }^{* * *}$ AND \\ STILIAN A. STOEV, ${ }^{* * * *}$ University of Michigan
}

\begin{abstract}
We develop classification results for max-stable processes, based on their spectral representations. The structure of max-linear isometries and minimal spectral representations play important roles. We propose a general classification strategy for measurable maxstable processes based on the notion of co-spectral functions. In particular, we discuss the spectrally continuous-discrete, the conservative-dissipative, and the positive-null decompositions. For stationary max-stable processes, the latter two decompositions arise from connections to nonsingular flows and are closely related to the classification of stationary sum-stable processes. The interplay between the introduced decompositions of max-stable processes is further explored. As an example, the Brown-Resnick stationary processes, driven by fractional Brownian motions, are shown to be dissipative.
\end{abstract}

Keywords: Max-stable process; classification; max-linear isometry; spectral representation; nonsingular flow; co-spectral function

2010 Mathematics Subject Classification: Primary 60G70

Secondary 60G10; 60G52; 37A50

\section{Introduction}

Max-stable processes have been studied extensively in the past 30 years. The works of Balkema and Resnick [2], de Haan [7], [8], de Haan and Pickands [9], Giné et al. [11], and Resnick and Roy [24], among many others, have led to a wealth of knowledge on max-stable processes. The seminal works of de Haan [8] and de Haan and Pickands [9] laid the foundations of the spectral representations of max-stable processes and established important structural results for stationary max-stable processes. Since then, however, while many authors focused on various important aspects of max-stable processes, the general theory of their representation and structural properties has not been thoroughly explored. At the same time, the structure and classification of sum-stable processes have been vigorously studied. Rosiński [26], building on the seminal works of Hardin [12], [13] about minimal representations, developed the important connection between stationary sum-stable processes and flows. This led to a number of important contributions on the structure of sum-stable processes (see, e.g. [21], [22], [27], [29], and [30]). There are relatively few results of this nature about the structure of max-stable processes, with the notable exceptions of de Haan and Pickands [9], Davis and Resnick [6], and the very recent works of Kabluchko et al. [16] and Kabluchko [14].

Our goal here is to develop representation and classification theory for max-stable processes, similar to that available for sum-stable processes. We are motivated by the strong similarities

Received 21 September 2009; revision received 20 May 2010.

* Postal address: Department of Statistics, The University of Michigan, 439 W. Hall, 1085 S. University, Ann Arbor, MI 48109-1107, USA.

** Email address: yizwang@umich.edu

*** Email address: sstoev@umich.edu 
between the spectral representations of sum- and max-stable processes. For max-stable processes, it turns out that the notion of minimal extremal integral representation plays a key role, as does the minimal integral representation for $\alpha$-stable processes (see [12], [13], [26], and [28]). Before we can fruitfully handle the minimal extremal integral representations, we should first thoroughly investigate the structure of max-linear isometries, also known as the pistons of de Haan and Pickands [9]. We refine and extend their work and give a brief summary in Section 3. A detailed and complete treatment is provided in [35]. Note that a recent work by Pipiras [20] provides a general framework to obtain minimal representations from any nonminimal representation for $\alpha$-stable processes. This framework could also be applied to max-stable processes.

In Section 4 we establish general classification results for max-stable processes using the developed theory of minimal spectral representations. In Section 4.1 we first show that essentially any max-stable process can be represented uniquely as the maximum of two independent components, characterized as spectrally continuous and spectrally discrete, respectively. In Section 4.2, for a large class of max-stable processes, we introduce the notion of co-spectral functions, which are invariant to the choice of spectral representations (up to a constant factor). This allows us to develop a general strategy for the classification of these processes, based on positive cones of co-spectral functions. As particular examples, we obtain the conservativedissipative and the positive-null decompositions.

Section 5 is devoted to the classification of stationary max-stable processes. As in the sum-stable case, the minimal representations allow us to associate a measurable nonsingular flow to every measurable stationary max-stable process. This correspondence enables us to apply existing ergodic theory results about the flow to characterize the max-stable process. The conservative-dissipative and positive-null decompositions introduced in Examples 4.3 and 4.4 are in fact motivated by the corresponding decompositions of the underlying flow. These two results are in close correspondence with the classifications of Rosiński [26] and Samorodnitsky [30] for sum-stable processes. As in Rosiński [26], we find that the class of stationary max-stable processes generated by dissipative flows is precisely the class of mixed moving maxima.

In Section 6 we apply the results in Section 5 to Brown-Resnick processes. We give simple necessary and sufficient conditions for a generalized Brown-Resnick stationary process to be a mixed moving maxima. This extends and complements the recent results of Kabluchko et al. [16]. In particular, if the Brown-Resnick process is generated by a fractional Brownian motion, then it is a mixed moving maxima.

The recent work of Kabluchko [14] established some classification results very similar to the ones appearing in Sections 4 and 5, using an association device between max- and sumstable processes. This association allows one to transfer existing classifications of sum-stable processes to the max-stable domain. It also clarifies the connection between these two classes of processes. Our results were obtained independently and by using rather different technical tools. The combination of the two approaches provides a clearer picture on the structure of max- and sum-stable processes as well as their interplay.

\section{Preliminaries}

The importance of max-stable processes stems from the fact that they arise in the limit of componentwise maxima of independent processes. It is well known that the univariate marginals of a max-stable process are necessarily extreme value distributions, i.e. up to rescaling and shift they are either Fréchet, Gumbel, or negative Fréchet. The dependence structure of the max- 
stable processes, however, can be quite intricate and it does not hinge on the extreme value type of the marginal distributions (see, e.g. Proposition 5.11 of [23]). Therefore, for convenience and without loss of generality, we will focus here on max-stable processes with Fréchet marginal distributions. Recall that a positive random variable $Z \geq 0$ has $\alpha$-Fréchet distribution, $\alpha>0$, if

$$
\mathrm{P}(Z \leq x)=\exp \left\{-\sigma^{\alpha} x^{-\alpha}\right\}, \quad x \in(0, \infty) .
$$

We will let $\|Z\|_{\alpha}:=\sigma>0$ denote for the scale coefficient of $Z$. It turns out that a stochastic process $\left\{X_{t}\right\}_{t \in T}$ with $\alpha$-Fréchet marginals is max-stable if and only if all positive max-linear combinations:

$$
\max _{1 \leq j \leq n} a_{j} X_{t_{j}} \equiv \bigvee_{1 \leq j \leq n} a_{j} X_{t_{j}} \quad \text { for all } a_{j}>0, t_{j} \in T, 1 \leq j \leq n,
$$

are $\alpha$-Fréchet random variables (see, e.g. [7] and [34]). This feature resembles the definition of Gaussian or, more generally, symmetric $\alpha$-stable (sum-stable) processes, where all finitedimensional linear combinations are univariate Gaussian or symmetric $\alpha$-stable, respectively (see, e.g. [31]). We will therefore refer to the max-stable processes with $\alpha$-Fréchet marginals as $\alpha$-Fréchet processes.

The seminal work of de Haan [8] provided convenient spectral representations for stochastically continuous $\alpha$-Fréchet processes in terms of functionals of Poisson point processes on $(0,1) \times(0, \infty)$. Here, we adopt the slightly more general, but essentially equivalent, approach of representing max-stable processes through extremal integrals with respect to random supmeasures (see [34]). We do so in order to emphasize the analogies with the well-developed theory of sum-stable processes (see, e.g. [31]).

Given a measure space $\left(S, \mathscr{B}_{S}, \mu\right)$ and $\alpha>0,\left\{M_{\alpha}(A)\right\}_{A \in B_{S}}$ is said to be an $\alpha$-Fréchet random sup-measure with control measure $\mu$, if (i) the $M_{\alpha}\left(A_{i}\right) \mathrm{s}$ are independent random variables for disjoint $A_{i} \in \mathcal{B}_{S}, 1 \leq i \leq n$, (ii) $M_{\alpha}(A)$ is $\alpha$-Fréchet with scale coefficient $\left\|M_{\alpha}(A)\right\|_{\alpha}=\mu(A)^{1 / \alpha}$, and (iii) for all disjoint $A_{i} \mathrm{~s}, i \in \mathbb{N}$, we have $M_{\alpha}\left(\bigcup_{i \in \mathbb{N}} A_{i}\right)=$ $\bigvee_{i \in \mathbb{N}} M_{\alpha}\left(A_{i}\right)$ almost surely.

We can then define the extremal integral of a nonnegative simple function $f(u):=\sum_{i=1}^{n} a_{i}$ $\times \mathbf{1}_{A_{i}}(u) \geq 0, A_{i} \in \mathscr{B}_{S}:$

$$
\int_{S}^{\mathrm{e}} f \mathrm{~d} M_{\alpha} \equiv \int_{S}^{\mathrm{e}} f(u) M_{\alpha}(\mathrm{d} u):=\bigvee_{1 \leq i \leq n} a_{i} M_{\alpha}\left(A_{i}\right) .
$$

Hence, ${ }^{\mathrm{e}} \int_{S} f \mathrm{~d} M_{\alpha}$ is an $\alpha$-Fréchet random variable with scale coefficient $\left(\int_{S} f^{\alpha} \mathrm{d} \mu\right)^{1 / \alpha}$. The definition of $\int_{S} f \mathrm{~d} M_{\alpha}$ can, by continuity in probability, be extended to integrands $f$ in the space of nonnegative, $L^{\alpha}$-integrable measurable functions $L_{+}^{\alpha}(S, \mu):=\left\{f \in L^{\alpha}(S, \mu): f \geq 0\right\}$. Here and in the sequel, we write $(S, \mu)=\left(S, \mathscr{B}_{S}, \mu\right)$ for simplicity. It turns out that the random variables $\int_{S} f_{j} \mathrm{~d} M_{\alpha}, 1 \leq j \leq n$, are independent, if and only if the $f_{j}$ s have pairwise disjoint supports $(\bmod \mu)$. Furthermore, the extremal integral is max-linear:

$$
\int_{S}^{\mathrm{e}}(a f \vee b g) \mathrm{d} M_{\alpha}=a \int_{S}^{\mathrm{e}} f \mathrm{~d} M_{\alpha} \vee b \int_{S}^{\mathrm{e}} g \mathrm{~d} M_{\alpha}
$$

for all $a, b>0$ and $f, g \in L_{+}^{\alpha}(S, \mu)$. For more details, see [34].

Now, for any collection of deterministic functions $\left\{f_{t}\right\}_{t \in T} \subset L_{+}^{\alpha}(S, \mu)$, we can construct the stochastic process

$$
X_{t}=\int_{S}^{\mathrm{e}} f_{t}(u) M_{\alpha}(\mathrm{d} u) \quad \text { for all } t \in T
$$


In view of the max-linearity of the extremal integrals and (2.1), the resulting process $X=$ $\left\{X_{t}\right\}_{t \in T}$ is $\alpha$-Fréchet. Furthermore, for any $n \in \mathbb{N}, x_{i}>0, t_{i} \in T, 1 \leq i \leq n$,

$$
\mathrm{P}\left(X_{t_{1}} \leq x_{1}, \ldots, X_{t_{n}} \leq x_{n}\right)=\exp \left\{-\int_{S}\left(\bigvee_{1 \leq i \leq n} x_{i}^{-1} f_{t_{i}}(u)\right)^{\alpha} \mu(\mathrm{d} u)\right\} .
$$

This shows that the deterministic functions, $\left\{f_{t}\right\}_{t \in T}$, completely characterize the finite-dimensional distributions of the process $X$. In general, if

$$
\left\{X_{t}\right\}_{t \in T} \stackrel{\mathrm{D}}{=}\left\{\int_{S}^{\mathrm{e}} f_{t} \mathrm{~d} M_{\alpha}\right\}_{t \in T}
$$

for some $\left\{f_{t}\right\}_{t \in T} \subset L_{+}^{\alpha}(S, \mu)$, we will say that the process $X$ has the extremal integral or spectral representation $\left\{f_{t}\right\}_{t \in T}$ over the space $L_{+}^{\alpha}(S, \mu)$. The $f_{t} \mathrm{~s}$ in (2.3) are also referred to as spectral functions of $X$.

Our goal in this paper is to characterize $\alpha$-Fréchet processes in terms of their spectral representations. Many $\alpha$-Fréchet processes of practical interest have tractable spectral representations, with $\left(S, \mathscr{B}_{S}, \mu\right)$ being a standard Lebesgue space (see, e.g. Appendix A of [22]). For example, a Polish space with $\sigma$-finite measure on its Borel sets is standard Lebesgue, and one often chooses $\left(S, \mathcal{B}_{S}, \mu\right)=\left([0,1], \mathcal{B}_{[0,1]}\right.$, Leb) in (2.3). As shown in Proposition 3.2 of [34], an $\alpha$-Fréchet process $X$ has representation (2.3) with $\left(S, \mathcal{B}_{S}, \mu\right)$ being standard Lebesgue, if and only if $X$ satisfies the following condition.

Condition S. There exists a countable subset $T_{0} \subseteq T$ such that, for every $t \in T$, we have $X_{t_{n}} \stackrel{\mathrm{P}}{\rightarrow} X_{t}$ for some $\left\{t_{n}\right\}_{n \in \mathbb{N}} \subset T_{0}$.

Note that without Condition $\mathrm{S}$, every max-stable process $X$ can still have a spectral representation as in $(2.3)$, but the space $(S, \mu)$ may not be standard Lebesgue (see Theorem 1 of [14]).

In the sequel, we focus only on the rich class of $\alpha$-Fréchet processes that satisfy Condition $\mathrm{S}$. This includes, for example, all measurable max-stable processes $X=\left\{X_{t}\right\}_{t \in T}$, indexed by a separable metric space $T$ (see Proposition 4.1 below). Recall that we write $(S, \mu)=\left(S, \mathcal{B}_{S}, \mu\right)$. The fact that $(S, \mu)$ is a standard Lebesgue space implies that the space of integrands $L_{+}^{\alpha}(S, \mu)$ is a complete and separable metric space with respect to the metric

$$
\rho_{\mu, \alpha}(f, g)=\int_{S}\left|f^{\alpha}-g^{\alpha}\right| \mathrm{d} \mu .
$$

This metric is natural to use when handling extremal integrals, since, as $n \rightarrow \infty$,

$$
\int_{S}^{\mathrm{e}} f_{n} \mathrm{~d} M_{\alpha} \stackrel{\mathrm{p}}{\rightarrow} \xi \quad \text { if and only if } \quad \rho_{\mu, \alpha}\left(f_{n}, f\right)=\int_{S}\left|f_{n}^{\alpha}-f^{\alpha}\right| \mathrm{d} \mu \rightarrow 0,
$$

where $\xi={ }^{\mathrm{e}} \int_{S} f \mathrm{~d} M_{\alpha}$ (see, e.g. [6] and [34]). In the sequel, we equip the space $L_{+}^{\alpha}(S, \mu)$ with the metric $\rho_{\mu, \alpha}$ and often write $\|f\|_{L_{+}^{\alpha}(S, \mu)}$ for $\left(\int_{S} f^{\alpha} \mathrm{d} \mu\right)^{1 / \alpha}$.

The max-linear (sub)spaces of functions in $L_{+}^{\alpha}(S, \mu)$ play a key role in the representation and characterization of max-stable processes. We say that $\mathcal{F}$ is a max-linear subspace of $L_{+}^{\alpha}(S, \mu)$ if $a f \vee b g \in \mathcal{F}$ for all $a, b>0$ and $f, g \in \mathcal{F}$, and $\mathcal{F} \subset L_{+}^{\alpha}(S, \mu)$ is closed with respect to the metric $\rho_{\mu, \alpha}$. In particular, provided a class of functions $\left\{f_{t}\right\}_{t \in T} \subset L_{+}^{\alpha}(S, \mu)$, we will frequently encounter the max-linear space $\mathcal{F}:=\overline{\mathrm{V}-\operatorname{span}}\left(f_{t}, t \in T\right)$, which is the smallest subset of 
$L_{+}^{\alpha}(S, \mu)$ that contains all max-linear combinations $\bigvee_{1 \leq i \leq n} a_{i} f_{t_{i}}, t_{i} \in T, a_{i}>0$, and is closed with respect to $\rho_{\mu, \alpha}$. A map $U$ from a max-linear subspace $\mathcal{F} \subset L_{+}^{\alpha}\left(S_{1}, \mu_{1}\right)$ to $L_{+}^{\alpha}\left(S_{2}, \mu_{2}\right)$ is said to be a max-linear isometry if (i) $U\left(a_{1} f_{1} \vee a_{2} f_{2}\right)=a_{1}\left(U f_{1}\right) \vee a_{2}\left(U f_{2}\right), \mu_{2}$-almost everywhere $\left(\mu_{2}\right.$-a.e.) for all $f_{1}, f_{2} \in \mathcal{F}$ and $a_{1}, a_{2} \geq 0$; and (ii) $\|U f\|_{L_{+}^{\alpha}\left(S_{2}, \mu_{2}\right)}=\|f\|_{L_{+}^{\alpha}\left(S_{1}, \mu_{1}\right)}$ for all $f \in \mathcal{F}$. A max-linear isometry $U$ is called a max-linear isomorphism if it is onto. For more details, see [34].

\section{Minimal representations}

We discuss here the notion of a minimal representation of an $\alpha$-Fréchet process, used extensively in the following sections. First, we remark that different spectral representations of the same process $\left\{X_{t}\right\}_{t \in T}$ can be related by max-linear isometries. Namely, assume that $\left\{f_{t}^{(i)}\right\}_{t \in T} \subset L_{+}^{\alpha}\left(S_{i}, \mu_{i}\right), i=1,2$, are two spectral representations for the $\alpha$-Fréchet process $X=\left\{X_{t}\right\}_{t \in T}$. By (2.2), for all $t_{j} \in \mathbb{R}, c_{j}>0,1 \leq j \leq n$, we have

$$
\mathrm{P}\left(X_{t_{j}} \leq c_{j}^{-1}, 1 \leq j \leq n\right)=\int_{S_{1}}\left(\bigvee_{j=1}^{n} c_{j} f_{t_{j}}^{(1)}\right)^{\alpha} \mathrm{d} \mu_{1}=\int_{S_{2}}\left(\bigvee_{j=1}^{n} c_{j} f_{t_{j}}^{(2)}\right)^{\alpha} \mathrm{d} \mu_{2} .
$$

We can thus define the natural max-linear isometry $U$ from $\mathcal{F}_{1}:=\overline{\nabla-\operatorname{span}}\left(\left\{f_{t}^{(1)}\right\}_{t \in T}\right)$ to $\mathcal{F}_{2}:=$ $\overline{\vee-\operatorname{span}}\left(\left\{f_{t}^{(2)}\right\}_{t \in T}\right)$ by

$$
U f_{t}^{(1)}:=f_{t}^{(2)} \quad \text { for all } t \in T .
$$

In the sequel, $U$ will be called the relating max-linear isometry of the two representations.

Our definition of a minimal representation is as in [26] (see also [9] and [13]). Other equivalent definitions have been investigated in [20] and [28]. This concept plays a key role in developing results on the structure of the relating max-linear isometry.

Definition 3.1. Given an $\alpha$-Fréchet process, a spectral representation $\left\{f_{t}\right\}_{t \in T} \subset L_{+}^{\alpha}(S, \mu)$ is said to be minimal if:

(i) $\operatorname{supp}\left\{f_{t}: t \in T\right\}=S, \mu$-a.e., where by $\operatorname{supp}\left\{f_{t}: t \in T\right\}$ we denote the minimal $(\bmod \mu)$ set $A \in \mathscr{B}_{S}$ such that $\mu\left\{s: f_{t}(s) \neq 0, s \in A^{\mathrm{c}}\right\}=0$ for every $t \in T$; and

(ii) for any $B \in \mathcal{B}_{S}$, there exists $A \in \rho\left(\left\{f_{t}: t \in T\right\}\right):=\sigma\left(\left\{f_{t} / f_{s}: t, s \in T\right\}\right)$ such that $\mu(A \Delta B)=0$. Here the ratios $f_{t} / f_{s}$ take values in $[0, \infty]$, with the conventions that $1 / 0=\infty$ and $0 / 0=0$.

We now state the main results on minimal representations. A complete treatment and the proofs can be found in [35]. The first result ensures the existence of minimal representations.

Theorem 3.1. Every $\alpha$-Fréchet process satisfying Condition $S$ has a minimal representation $\left\{f_{t}\right\}_{t \in T} \subset L_{+}^{\alpha}(S, \mu)$, where $(S, \mu)$ is a standard Lebesgue space. Namely,

$$
\left\{X_{t}\right\}_{t \in T} \stackrel{\mathrm{D}}{=}\left\{\int_{S}^{\mathrm{e}} f_{t}(s) M_{\alpha}(\mathrm{d} s)\right\}_{t \in T},
$$

where $M_{\alpha}$ is the $\alpha$-Fréchet random sup-measure with control measure $\mu$.

The next result shows that a general spectral representation can be related to a minimal one via point mappings. 
Theorem 3.2. Let $\left\{g_{t}\right\}_{t \in T} \subset L_{+}^{\alpha}\left(S_{1}, \mu_{1}\right)$ and $\left\{f_{t}\right\}_{t \in T} \subset L_{+}^{\alpha}\left(S_{2}, \mu_{2}\right)$ be two spectral representations of an $\alpha$-Fréchet process $\left\{X_{t}\right\}_{t \in T}$. Let $U$ be the relating max-linear isometry of $\left\{g_{t}\right\}_{t \in T}$ and $\left\{f_{t}\right\}_{t \in T}$. If $\left\{g_{t}\right\}_{t \in T}$ is minimal and $\left\{f_{t}\right\}_{t \in T}$ is arbitrary, then

(i) $U$ can be uniquely extended to $L_{+}^{\alpha}\left(S_{1}, \mu_{1}\right)$;

(ii) $U$ can be represented by measurable functions $\Phi: S_{2} \rightarrow S_{1}$ and $h: S_{2} \rightarrow \mathbb{R}_{+} \backslash\{0\}$, such that $\Phi$ is onto, and the following statements hold:

$$
f_{t}(s)=U g_{t}(s)=h(s)\left(g_{t} \circ \Phi\right)(s) \quad \mu_{2} \text {-a.e. for all } t \in T
$$

and

$$
\mu_{1}=\mu_{h} \circ \Phi^{-1},
$$

where $\mu_{h}(\mathrm{~d} s)=h(s)^{\alpha} \mu_{2}(\mathrm{~d} s)$. Moreover, $\Phi$ is unique modulo $\mu_{2}$.

We will also consider minimal representations with standardized support. A similar idea of standardization was mentioned in [25].

Definition 3.2. A minimal representation $\left\{f_{t}\right\}_{t \in T} \subset L_{+}^{\alpha}(S, \mu)$ has standardized support if, up to $\mu$-null sets,

(i) $S \subset(0,1) \cup \mathbb{N}$;

(ii) $S \cap(0,1)=\varnothing$ or $(0,1)$ and $\left.\mu\right|_{S \cap(0,1)}$ is the Lebesgue measure;

(iii) $S \cap \mathbb{N}=\varnothing, \mathbb{N}$ or $\{1, \ldots, N\}$, where $N \in \mathbb{N}$ and $\left.\mu\right|_{S \cap \mathbb{N}}$ is the counting measure.

Let $\left(S_{I, N}, \lambda_{I, N}\right)$ denote the standard support with $I=0$ or 1 respectively according to the two cases in (ii), and $N=0, N=\infty$, or $N \in \mathbb{N}$ respectively according to the three cases in (iii), e.g. $S_{0, \infty}=\mathbb{N}$ and $S_{1, N}=(0,1) \cup\{1, \ldots, N\}$.

The next two corollaries refine Theorems 3.1 and 3.2, respectively.

Corollary 3.1. The minimal spectral functions $\left\{f_{t}\right\}_{t \in T} \subset L_{+}^{\alpha}(S, \mu)$ in Theorem 3.1 can be chosen to have standardized support.

For convenience, write $S^{I}:=S_{I, N} \cap(0,1)$ and $S^{N}:=S_{I, N} \cap \mathbb{N}$, and, for all $f \in L_{+}^{\alpha}(S, \mu)$, write $f^{I}:=f \mathbf{1}_{S^{I}}$ and $f^{N}:=f \mathbf{1}_{S^{N}}$. The following corollary shows that the standardized support does not depend on the choice of the minimal representation.

Corollary 3.2. Let $\left\{f_{t}\right\}_{t \in T}$ and $\left\{g_{t}\right\}_{t \in T}$ be two minimal representations of an $\alpha$-Fréchet process $\left\{X_{t}\right\}_{t \in T}$ with standardized supports $\left(S_{I_{i}, N_{i}}, \lambda_{I_{i}, N_{i}}\right), i=1,2$, respectively. Then

$$
I_{1}=I_{2}=I \text { and } N_{1}=N_{2}=N
$$

Moreover, the relating max-linear isometry $U$ of these representations has the form

$$
U f_{t}^{I}=\left(\frac{\mathrm{d}\left(\lambda \circ \Phi_{I}\right)}{\mathrm{d} \lambda}\right)^{1 / \alpha}\left(f_{t}^{I} \circ \Phi_{I}\right)=g_{t}^{I} \quad \lambda \text {-a.e. and } U f_{t}^{N}=f_{t}^{N} \circ \Phi_{N}=g_{t}^{N}
$$

for all $t \in T$, where $\lambda$ is the Lebesgue measure on $(0,1), \Phi_{I}: S^{I} \rightarrow S^{I}$ and $\Phi_{N}: S^{N} \rightarrow S^{N}$ are bijections, and $\Phi_{I}$ is bi-measurable. 
Remark 3.1. We briefly sketch how to show Theorem 3.2, since (3.2) and (3.3) play a crucial role in the sequel.

First, we can represent the relating max-linear isometry $U$ in (3.1) in terms of a regular set isomorphism $\mathbb{T}$ (see Definition 3.2 of [35]) from $\left(S_{1}, \rho\left(\mathcal{F}_{1}\right)\right)$ to $\left(S_{2}, \rho\left(\mathcal{F}_{2}\right)\right.$ ). The regular set isomorphism $\mathbb{T}$ is a set mapping, which induces a function mapping for all $\rho\left(\widetilde{F}_{1}\right)$-measurable functions $f$ (see, e.g. [10, pp. 452-454]). Next, we can extend $U$ to a max-linear isometry $\bar{U}$ on a larger subspace of $L_{+}^{\alpha}\left(S_{1}, \mu_{1}\right)$, consisting of all functions in the product form $r f$, where $r$ is $\rho\left(\mathcal{F}_{1}\right)$-measurable, $f \in \mathcal{F}_{1}$, and $r f \in L_{+}^{\alpha}\left(S_{1}, \mu_{1}\right)$. The mapping $\bar{U}$ has the following form:

$$
\bar{U}(r f)=(\mathbb{T} r)(U f) \text { for all } r \in \rho\left(\mathcal{F}_{1}\right), f \in \mathscr{F}_{1} .
$$

Under the minimality assumption, Theorem 32.5 of [32] implies that $\mathbb{T}$ is generated by a point mapping from $S_{2}$ to $S_{1}$. This will eventually yield Theorem 3.2 (see the proof of Theorem 4.2 of [35]).

\section{Classification of $\alpha$-Fréchet processes}

We now apply the abstract results on max-linear isometries and minimal representations to classify $\alpha$-Fréchet processes. We start by dealing with the measurability of spectral functions $f_{t}(s)$, seen as functions from $(T, S)$ to $\mathbb{R}_{+}$. Suppose that $\mathcal{T}$ is a $\sigma$-algebra on $T$. We say that the spectral representation $\left\{f_{t}(s)\right\}_{t \in T} \subset L_{+}^{\alpha}(S, \mu)$ is jointly measurable if the mapping $(t, s) \mapsto f_{t}(s)$ is measurable with respect to the product $\sigma$-algebra $\mathcal{T} \otimes \mathcal{B}_{S}:=\sigma\left(\mathcal{T} \times \mathcal{B}_{S}\right)$. By Theorem 18.1 of [3], $f$. (s) is $\mathcal{T}$-measurable for all $s \in S$. The following result clarifies the connection between the joint measurability of the spectral functions $f_{t}(s)$ and the measurability of its corresponding $\alpha$-Fréchet process. The proof is given in Appendix A.

Proposition 4.1. Let $(S, \mu)$ be a standard Lebesgue space, and let $M_{\alpha}(\alpha>0)$ be an $\alpha$-Fréchet random sup-measure on $S$ with control measure $\mu$. Suppose that $\left(T, \rho_{T}\right)$ is a separable metric space and that $\mathcal{T}$ is the Borel $\sigma$-algebra.

(i) Let $X=\left\{X_{t}\right\}_{t \in T}$ have a spectral representation $\left\{f_{t}\right\}_{t \in T} \subset L_{+}^{\alpha}(S, \mu)$ as in (2.3). Then, $X$ has a measurable modification if and only if $\left\{f_{t}(s)\right\}_{t \in T}$ has a jointly measurable modification, i.e. there exists a $\left(\mathcal{T} \otimes \mathcal{B}_{S}\right)$-measurable mapping $(s, t) \mapsto g_{t}(s)$, such that $f_{t}(s)=g_{t}(s)$, $\mu$-a.e. for all $t \in T$.

(ii) If an $\alpha$-Fréchet process $\left\{X_{t}\right\}_{t \in T}$ has a measurable modification then it satisfies Condition $S$, and, hence, it has a representation as in (2.3).

From now on, we assume that $\left(T, \rho_{T}\right)$ is a separable metric space and that $\mathcal{T}$ is the Borel $\sigma$-algebra. By Proposition 4.1, any measurable $\alpha$-Fréchet process $\left\{X_{t}\right\}_{t \in T}$ always has a jointly measurable spectral representation and satisfies Condition $\mathrm{S}$.

\subsection{Continuous-discrete decomposition}

Consider an $\alpha$-Fréchet process $X=\left\{X_{t}\right\}_{t \in T}$, which has a minimal representation with standardized support $\left\{g_{t}\right\}_{t \in T} \subset L_{+}^{\alpha}\left(S_{I, N}, \lambda_{I, N}\right)$. By Corollary 3.2, the support $\left(S_{I, N}, \lambda_{I, N}\right)$ is unique. We therefore call $S_{I, N}$ the standardized support of $X$ and focus on the continuous and discrete parts, respectively. We can write

$$
\left\{X_{t}\right\}_{t \in T} \stackrel{\mathrm{D}}{=}\left\{X_{t}^{I} \vee X_{t}^{N}\right\}_{t \in T},
$$

where $X_{t}^{I}:={ }^{\mathrm{e}} \int_{S^{I}} g_{t}^{I}(s) M_{\alpha}(\mathrm{d} s)$ and $X_{t}^{N}:={ }^{\mathrm{e}} \int_{S^{N}} g_{t}^{N}(s) M_{\alpha}(\mathrm{d} s)$ are two independent $\alpha$-Fréchet processes. 
We will provide next a classification rule, which allows us to obtain decomposition (4.1) for essentially any jointly measurable spectral representation $\mathcal{F}=\left\{f_{t}\right\}_{t \in T} \subset L_{+}^{\alpha}(S, \mu)$. Recall that $X$ satisfies Condition S, i.e. there exists a countable subset $T_{0} \in T$ and, for all $t \in T$, there exist $\left\{t_{n}\right\}_{n \in \mathbb{N}} \subset T_{0}$ such that $X_{t_{n}} \rightarrow X_{t}$ in probability as $n \rightarrow \infty$. This implies that $f_{t_{n_{k}}} \rightarrow f_{t}, \mu$-a.e. for some subsequence $n_{k} \rightarrow \infty$ as $k \rightarrow \infty$ (see Theorem 2.1 of [34]). In this way, the spectral functions $\mathcal{F}$ are determined by $\mathcal{F}_{T_{0}}=\left\{f_{t}\right\}_{t \in T_{0}}$, and we can show that $\rho\left(\mathcal{F}_{T_{0}}\right) \sim \rho(\mathcal{F})$, i.e. for all $A \in \rho(\mathcal{F})$, there exists $\widetilde{A} \in \rho\left(\mathcal{F}_{T_{0}}\right)$ such that $\mu(A \triangle \widetilde{A})=0$. From now on, we fix $T_{0}$ and our classification rule depends only on $\mathcal{F}_{T_{0}}$.

First, define an equivalence relation on $S$ as follows:

$$
s \sim r \text { if } f_{t}(s)=q_{s, r} f_{t}(r) \quad \text { for all } t \in T_{0} \text { and some constant } q_{s, r}>0 .
$$

Let $[s]=\{r: r \in S, r \sim s\}$ denote the equivalence class of $s$. To see that $[s]$ is measurable, it suffices to find a measurable function $Q_{\mathcal{F}_{T_{0}}}(s, r)$ such that $s \sim r$ if and only if $Q_{\mathcal{F}_{T_{0}}}(s, r)=0$. A possible choice is

$$
Q_{\mathcal{F}_{T_{0}}}(s, r)=\sum_{t_{1}, t_{2} \in T_{0}} \mathbf{1}\left\{\frac{f_{t_{1}}(s)}{f_{t_{1}}(r)} \neq \frac{f_{t_{2}}(s)}{f_{t_{2}}(r)}\right\}+\sum_{t \in T_{0}} \mathbf{1}\left\{\frac{f_{t}(s)}{f_{t}(r)}=\infty \text { or } \frac{f_{t}(r)}{f_{t}(s)}=\infty\right\},
$$

where $0 / 0=0$ and $1 / 0=\infty$. The equivalence relation (4.2) will help us obtain the desired decomposition. The idea is that the union of all $[s]$ with $\mu([s])>0$ corresponds to the spectrally discrete component. Indeed, observe that (4.2) implies that, for all $t_{1}, t_{2} \in T_{0}$,

$$
\frac{f_{t_{1}}(s)}{f_{t_{2}}(s)}=\frac{f_{t_{1}}(r)}{f_{t_{2}}(r)} \quad \text { equals a constant in }[0, \infty] \text { for all } s \in[r] .
$$

This shows that the atoms of $\rho\left(\mathcal{F}_{T_{0}}\right)$ correspond to classes $[s]$ with $\mu([s])>0$. This suggests defining the spectrally discrete support of $\left\{f_{t}\right\}_{t \in T}$ by

$$
\widetilde{S}^{N}:=\bigcup_{\{s: \mu([s])>0\}}[s]=\left\{s: \int_{S} \mathbf{1}_{\left\{Q_{\mathcal{F}_{T}}(s, r)=0\right\}} \mu(\mathrm{d} r)>0\right\}
$$

and the spectrally continuous support by $\widetilde{S}^{I}:=S \backslash \widetilde{S}^{N}=\{s: \mu([s])=0\}$.

Since the measure $\mu$ is $\sigma$-finite, there are at most countably many classes [s] with $\mu([s])>0$. Therefore, their union $\widetilde{S}^{N}$ is measurable and so is $\widetilde{S}^{I}$. Now, we can consider decomposition (4.1) with

$$
X_{t}^{I}:=\int_{\widetilde{S}^{I}}^{\mathrm{e}} f_{t}(s) M_{\alpha}(\mathrm{d} s) \quad \text { and } \quad X_{t}^{N}:=\int_{\widetilde{S}^{N}} f_{t}(s) M_{\alpha}(\mathrm{d} s) \quad \text { for all } t \in T .
$$

Theorem 4.1. Let $\left\{X_{t}\right\}_{t \in T}$ be an $\alpha$-Fréchet process with jointly measurable representation $\left\{f_{t}\right\}_{t \in T} \subset L_{+}^{\alpha}(S, \mu)$. Then,

(i) decomposition (4.1) with components defined by (4.5) is unique in distribution; and

(ii) the processes $X^{I}=\left\{X_{t}^{I}\right\}_{t \in T}$ and $X^{N}=\left\{X_{t}^{N}\right\}_{t \in T}$ are independent, and their minimal representations can be chosen to have standardized supports $S^{I}$ and $S^{N}$, respectively.

Proof. See Appendix A.

The processes $\left\{X_{t}^{I}\right\}_{t \in T}$ and $\left\{X_{t}^{N}\right\}_{t \in T}$ will be referred to as the spectrally continuous and spectrally discrete components of $X$, respectively. 
Remark 4.1. The choice of the appropriate set $T_{0}$ in (4.2) is delicate. Other natural choices would either lead to inconsistent classification rules, or they would not yield the desired continuous-discrete decomposition. Indeed, suppose first that we replace 'for all $t \in T_{0}$ ' by 'for all $t \in T$ ' in (4.2) and consider $f_{t}(s)=1$ with $S=T=(0,1), \mu=\lambda=$ Leb. Clearly, we have $\widetilde{S}^{N}=(0,1)$. Let, however, $\widetilde{f}_{t}(s)=f_{t}(s)+\mathbf{1}_{\{t=s\}}$ be a modification of $f_{t}(s)$. Now, by replacing $T_{0}$ with $T$ and $\mathcal{F}_{T_{0}}$ with $\widetilde{\mathcal{F}}_{T}=\left\{\widetilde{f}_{t}: t \in T\right\}$ in (4.4), we obtain $\widetilde{S}^{N}=\varnothing$, since in this case $[s]=\{s\}$ for all $s \in(0,1)$. Hence, this classification rule is inconsistent because it depends on the choice of the (equivalent) spectral representation.

Next, suppose that 'for all $t \in T_{0}$ ' is replaced by 'for $\lambda$-almost all $t \in T$ ' in (4.2). Consider $f_{t}(s)=1+s \mathbf{1}_{\{t=1 / 2\}}$ with $S=T=(0,1)$ and $\mu=\lambda=$ Leb. This representation is minimal with standardized support and, in particular, with trivial spectrally discrete component. The modified classification rule would yield, however, $\widetilde{S}^{N}=(0,1)$, since $[s]=(0,1)$ for all $s \in(0,1)$. To obtain the continuous-discrete decomposition, in this case it is important to include the point $t=\frac{1}{2}$ in the index set used in (4.2).

The following two examples illustrate typical spectrally discrete and spectrally continuous processes.

Example 4.1. Let $Z_{i}, i \in \mathbb{N}$, be independent standard $\alpha$-Fréchet variables, and let $f_{t}(i) \geq 0$, $t \in T$, be such that $\sum_{i \in \mathbb{N}} f_{t}^{\alpha}(i)<\infty$ for all $t \in T$. The $\alpha$-Fréchet process

$$
X_{t}:=\bigvee_{i \in \mathbb{N}} f_{t}(i) Z_{i} \equiv \int_{\mathbb{N}}^{\mathrm{e}} f_{t} \mathrm{~d} M_{\alpha}, \quad t \in T,
$$

is spectrally discrete.

Example 4.2. Consider the well-known $\alpha$-Fréchet extremal process $(\alpha>0)$

$$
\left\{X_{t}\right\}_{t \in \mathbb{R}_{+}} \stackrel{\mathrm{D}}{=}\left\{\int_{\mathbb{R}_{+}}^{\mathrm{e}} \mathbf{1}_{(0, t]}(u) M_{\alpha}(\mathrm{d} u)\right\}_{t \in \mathbb{R}_{+}},
$$

where $M_{\alpha}$ has the Lebesgue control measure on $\mathbb{R}_{+}$. The process $X=\left\{X_{t}\right\}_{t \in \mathbb{R}_{+}}$can be viewed as the max-stable counterpart to a sum-stable Lévy process. The $\alpha$-Fréchet extremal process $X$ is spectrally continuous.

\subsection{Classification via co-spectral functions}

Here we present a characterization of $\alpha$-Fréchet processes based on a different point of view. Namely, instead of focusing on the spectral functions $s \mapsto f_{t}(s)$, we now consider the co-spectral functions $t \mapsto f_{t}(s)$, which are functions of $t$, with $s$ fixed. Let $\lambda$ now be a $\sigma$-finite Borel measure on $(T, \mathcal{T})$ and view each $f(s)$ as an element of the classes $L_{+}^{0}(T, \mathcal{T}, \lambda)$ of nonnegative $\mathcal{T}$-measurable functions, identified with respect to equality $\lambda$-a.e.

We start with some intuition. Observe that, for the two spectral representations of the same $\alpha$-Fréchet process $\left\{X_{t}\right\}_{t \in T}$ as in Theorem 3.2, relation (3.2) implies that the co-spectral functions $t \mapsto f_{t}(s)$ are proportional to $g_{t} \circ \Phi(s)$. This motivates us to classify the process $X$ according to, roughly speaking, the 'shape' of the co-spectral functions $f$. $(s)$. To explore this idea, it is natural to consider positive cones, as $g . \circ \Phi(s)$ and $f(s)$ are in the same positive cone. Recall that a set $\mathcal{P} \subset L_{+}^{0}(T, \mathcal{T}, \lambda)$ is said to be a positive cone if $c \mathcal{P} \subset \mathcal{P}$ for all $c \geq 0$. Two cones $\mathcal{P}_{1}$ and $\mathcal{P}_{2}$ are disjoint if $\mathcal{P}_{1} \cap \mathcal{P}_{2}=\{0\}$.

We propose a general strategy for classification of $\alpha$-Fréchet processes, based on any collection of disjoint positive cones $\mathcal{P}_{j} \subset L_{+}^{0}(T, \mathcal{T}, \lambda), 1 \leq j \leq n$. For any $\alpha$-Fréchet process 
$X=\left\{X_{t}\right\}_{t \in T}$ with jointly measurable representation of $\left\{f_{t}(s)\right\}_{t \in T} \subset L_{+}^{\alpha}(S, \mu)$ with full support (i.e. $\operatorname{supp}(\mathcal{F})=S$ ), we say that the representation has a co-spectral decomposition with respect to $\left\{\mathcal{P}_{j}\right\}_{1 \leq j \leq n}$ if there exist measurable sets $S^{(j)}, 1 \leq j \leq n$, such that

$$
S^{(j)} \subset\left\{s \in S: f .(s) \in \mathcal{P}_{j}\right\}, \quad 1 \leq j \leq n, \quad \text { and } \quad \mu\left(S \backslash \bigcup_{j=1}^{n} S^{(j)}\right)=0 .
$$

The sets $S^{(j)}, 1 \leq j \leq n$, are modulo $\mu$ disjoint. Indeed, let $A:=\{s \in S: f .(s) \equiv 0\}$ and note that $\mu(A)=0$ by the fact that $\operatorname{supp}\left\{f_{t}, t \in T\right\}=S$ modulo $\mu$ and Fubini's theorem. Since $\mathcal{P}_{j} \cap \mathcal{P}_{k}=\{0\}$, we have $S^{(j)} \cap S^{(k)}=A$ for all $1 \leq j \neq k \leq n$. That is, the space $S$ is partitioned into $n$ modulo $\mu$ disjoint components:

$$
S=S^{(1)} \cup \cdots \cup S^{(n)} \quad \bmod \mu, \quad \text { with } \mu\left(S^{(j)} \cap S^{(k)}\right)=0, \quad j \neq k .
$$

This yields the decomposition

$$
\left\{X_{t}\right\}_{t \in T} \stackrel{\mathrm{D}}{=}\left\{X_{t}^{(1)} \vee \cdots \vee X_{t}^{(n)}\right\}_{t \in T},
$$

with

$$
X_{t}^{(j)}:=\int_{S^{(j)}}^{\mathrm{e}} f_{t}(s) M_{\alpha}(\mathrm{d} s) \quad \text { for } 1 \leq j \leq n \text { and all } t \in T .
$$

The next result shows that decomposition is invariant with respect to the choice of spectral representation.

Theorem 4.2. Suppose that $\left\{\mathcal{P}_{j}\right\}_{1 \leq j \leq n}$ are disjoint positive cones in $L_{+}^{0}(T, \mathcal{T}, \lambda)$. For any $\alpha$ Fréchet process $\left\{X_{t}\right\}_{t \in T}$ with jointly measurable spectral representation $\left\{f_{t}\right\}_{t \in T} \subset L_{+}^{\alpha}(S, \mu)$, suppose that $\left\{f_{t}\right\}_{t \in T}$ has a co-spectral decomposition with respect to $\left\{\mathcal{P}_{j}\right\}_{1 \leq j \leq n}$. Then,

(i) decomposition (4.9) is unique in distribution; and

(ii) the components $\left\{X_{t}^{(j)}\right\}_{t \in T}, 1 \leq j \leq n$, are independent $\alpha$-Fréchet processes.

Proof. See Appendix A.

In the special case in which $n=1$, Theorem 4.2 yields the following result.

Corollary 4.1. Let $X=\left\{X_{t}\right\}_{t \in T}$ be an $\alpha$-Fréchet process with two jointly measurable representations $\left\{f_{t}^{(i)}(s)\right\}_{t \in T} \subset L_{+}^{\alpha}\left(S_{i}, \mu_{i}\right), i=1,2$. Consider a positive cone $\mathcal{P} \subset L_{+}^{0}(T, \mathcal{T}, \lambda)$. If $f^{(1)}(s) \in \mathscr{P}$ for $\mu_{1}$-almost all $s \in S_{1}$ then $f^{(2)}(s) \in \mathcal{P}$ for $\mu_{2}$-almost all $s \in S_{2}$.

Corollary 4.1 can be used to distinguish between various $\alpha$-Fréchet processes in terms of their co-spectral functions. For example, any measurable representation of the $\alpha$-Fréchet extremal process in (4.6) should involve simple indicator-type co-spectral functions with one jump down to 0 . The next result shows another application of Corollary 4.1.

Corollary 4.2. Consider the moving maxima $\alpha$-Fréchet random fields

$$
\left\{X_{t}\right\}_{t \in \mathbb{R}^{d}} \stackrel{\mathrm{D}}{=}\left\{\int_{\mathbb{R}^{d}}^{\mathrm{e}} f(t-s) M_{\alpha}(\mathrm{d} s)\right\}_{t \in \mathbb{R}^{d}} \text { and }\left\{Y_{t}\right\}_{t \in \mathbb{R}^{d}} \stackrel{\mathrm{D}}{=}\left\{\int_{\mathbb{R}^{d}}^{\mathrm{e}} g(t-s) M_{\alpha}(\mathrm{d} s)\right\}_{t \in \mathbb{R}^{d}},
$$

with $d \in \mathbb{N}$ and $f, g \in L_{+}^{\alpha}\left(\mathbb{R}^{d}, \lambda\right)$, where $\lambda$ is the Lebesgue measure. Here $M_{\alpha}$ is an $\alpha$-Fréchet random sup-measure on $\mathbb{R}^{d}$ with the Lebesgue control measure. We have $\left\{X_{t}\right\}_{t \in T} \stackrel{\mathrm{D}}{=}\left\{Y_{t}\right\}_{t \in T}$ if and only if, for some fixed $\tau \in \mathbb{R}^{d}, g(\cdot)=f(\cdot+\tau)$ in $L_{+}^{\alpha}\left(\mathbb{R}^{d}, \lambda\right)$. 
Proof. The 'if' part is trivial. To prove the 'only if' part, introduce the cone $\mathcal{P}_{f}=\{c f(\cdot+$ $\left.\tau), c \geq 0, \tau \in \mathbb{R}^{d}\right\}$. Recall that $f$ is viewed as an equivalence class (modulo $\lambda$ ) of functions in $L_{+}^{0}(T, \mathcal{T}, \lambda)$. Corollary 4.1 implies that $g(\cdot) \in \mathcal{P}_{f}$, and, hence, $g(\cdot)=c f(\cdot+\tau)$ in $L_{0}^{+}(T, \mathcal{T}, \lambda)$ for some $\tau \in \mathbb{R}^{d}$. Since

$$
\left\|X_{0}\right\|_{\alpha}^{\alpha}=\int_{\mathbb{R}^{d}} g^{\alpha}(x) \mathrm{d} x=\int_{\mathbb{R}^{d}} f^{\alpha}(x) \mathrm{d} x,
$$

it follows that $c=1$. This completes the proof.

Theorem 4.2 is a general result in the sense that the cones $\left\{\mathcal{P}_{j}\right\}_{1 \leq j \leq n}$ may be associated with various properties of the co-spectral functions $t \mapsto f_{t}(s)$ of the process $X$. If $T \equiv \mathbb{R}^{d}, d \geq 1$, for example, we can consider the cones of co-spectral functions that have differentiable, continuous, integrable, or $\beta$-Hölder continuous versions. Note that here we see the co-spectral functions as elements in $L_{+}^{0}(T, \mathcal{T}, \lambda)$. Every choice of cones leads to different types of classification for measurable $\alpha$-Fréchet processes or fields $X=\left\{X_{t}\right\}_{t \in T}$. We conclude this section by giving two important examples of classifications, motivated by existing results in the literature on sum-stable processes.

Remark 4.2. Note that, instead of (4.7), we may want to set $S^{(j)}:=\left\{s: f .(s) \in \mathcal{P}_{j}\right\}, 1 \leq$ $j \leq n$. However, for certain cones, the measurability of the so-defined $S^{(j)}$ s will be hard to prove. See Example 4.4 below.

Example 4.3. (Conservative-dissipative decomposition.) Let $X=\left\{X_{t}\right\}_{t \in T}$ be an $\alpha$-Fréchet process with jointly measurable representation $\left\{f_{t}(s)\right\}_{t \in T} \subset L_{+}^{\alpha}(S, \mu)$. Consider the following cones:

$$
\begin{aligned}
& \mathcal{P}_{C}=\left\{h: h \in L_{+}^{0}(T, \mathcal{T}, \lambda), \int_{T} h^{\alpha}(t) \lambda(\mathrm{d} t)=\infty\right\} \cup\{0\}, \\
& \mathcal{P}_{D}=\left\{h: h \in L_{+}^{0}(T, \mathcal{T}, \lambda), \int_{T} h^{\alpha}(t) \lambda(\mathrm{d} t)<\infty\right\},
\end{aligned}
$$

and define

$$
C:=\left\{s: s \in S, f .(s) \in \mathcal{P}_{C}\right\} \quad \text { and } \quad D:=\left\{s: s \in S, f .(s) \in \mathcal{P}_{D}\right\} .
$$

By Fubini's theorem, the sets $C$ and $D$ are both measurable. This partition of $S$ yields the decomposition

$$
\left\{X_{t}\right\}_{t \in T} \stackrel{\mathrm{D}}{=}\left\{X_{t}^{C} \vee X_{t}^{D}\right\}_{t \in T},
$$

where $X^{C}=\left\{X_{t}^{C}\right\}_{t \in T}$ and $X^{D}=\left\{X_{t}^{D}\right\}_{t \in T}$ are defined as

$$
X_{t}^{C}=\int_{C}^{\mathrm{e}} f_{t} \mathrm{~d} M_{\alpha} \quad \text { and } \quad X_{t}^{D}=\int_{D}^{\mathrm{e}} f_{t} \mathrm{~d} M_{\alpha} \quad \text { for all } t \in T .
$$

Here $M_{\alpha}$ is an $\alpha$-Fréchet random sup-measure with control measure $\mu$.

By Theorem 4.2, decomposition (4.11) does not depend on the choice of representation. The components $X^{C}$ and $X^{D}$ of $X$ are independent and they are called the conservative and dissipative parts of $X$, respectively. Decomposition (4.11) is referred to as the conservativedissipative decomposition. 
Example 4.4. (Positive-null decomposition.) Following Samorodnitsky [30], consider $T=\mathbb{R}$ or $\mathbb{Z}$. Introduce the class $\mathcal{W}$ of positive weight functions $w: T \rightarrow \mathbb{R}_{+}$:

$$
\mathcal{W}:=\left\{w: \int_{T} w(t) \lambda(\mathrm{d} t)=\infty, w(t) \text { and } w(-t) \text { are nondecreasing on } T \cap(0, \infty)\right\} .
$$

Now we consider the cone

$$
\mathcal{P}_{\text {pos }}:=\left\{h \in L_{+}^{0}(T, \mathcal{T}, \lambda): \int_{T} w(t) h^{\alpha}(t) \lambda(\mathrm{d} t)=\infty \text { for all } w \in \mathcal{W}\right\} \cup\{0\}
$$

and its complement cone $\mathcal{P}_{\text {null }}:=\{0\} \cup\left(L_{+}^{0}(T, \mathcal{T}, \lambda) \backslash \mathcal{P}_{\text {pos }}\right)$.

This choice of cones yields the decomposition

$$
\left\{X_{t}\right\}_{t \in T} \stackrel{\mathrm{D}}{=}\left\{X_{t}^{\mathrm{pos}} \vee X_{t}^{\text {null }}\right\}_{t \in T},
$$

where

$$
X_{t}^{\mathrm{pos}}:=\int_{P}^{\mathrm{e}} f_{t}(s) M_{\alpha}(\mathrm{d} s) \quad \text { and } \quad X_{t}^{\mathrm{null}}:=\int_{N}^{\mathrm{e}} f_{t}(s) M_{\alpha}(\mathrm{d} s) \quad \text { for all } t \in T,
$$

with $P$ and $N$, measurable subsets of $S$, satisfying $\mu(P \cap N)=0, \mu(S \backslash(P \cup N))=0$, and

$$
f .(s) \in \mathcal{P}_{\text {pos }} \quad \text { for all } s \in P \quad \text { and } \quad f .(s) \in \mathcal{P}_{\text {null }} \quad \text { for all } s \in N .
$$

The components $X^{\mathrm{pos}}=\left\{X_{t}^{\mathrm{pos}}\right\}_{t \in T}$ and $X^{\text {null }}=\left\{X_{t}^{\text {null }}\right\}_{t \in T}$ in (4.14) are said to be the positive and null components of the process $X$, respectively. By Theorem 4.2, decomposition (4.13) does not depend on the choice of the measurable representation $\left\{f_{t}(s)\right\}_{t \in T} \subset L_{+}^{\alpha}(S, \mu)$. It is referred to as the positive-null decomposition.

Note that, a technical difference between this example and Example 4.3 is that, to the best of the authors' knowledge, it is not clear how to show whether the set $\widetilde{P}:=\left\{s: f .(s) \in \mathcal{P}_{\text {pos }}\right\}$ is (or not) measurable, even when $f_{t}(s)$ is jointly measurable.

In the following section, we will study the above decompositions in more detail, for the case of stationary max-stable processes.

\section{Classification of stationary $\alpha$-Fréchet processes}

In this section we focus on stationary, measurable max-stable processes $X=\left\{X_{t}\right\}_{t \in T}$, where $T=\mathbb{R}$ or $T=\mathbb{Z}$ is equipped with the Lebesgue or the counting measure $\lambda$, respectively. In this case, the process $X$ can be associated with a nonsingular flow. Therefore, as in the symmetric $\alpha$-stable case, the ergodic theoretic properties of the flow yield illuminating structural results.

\subsection{Nonsingular flows associated with max-stable processes}

Following Rosiński [26], we recall some notions from ergodic theory.

Definition 5.1. A family of functions $\phi=\left\{\phi_{t}\right\}_{t \in T}, \phi_{t}: S \rightarrow S$ for all $t \in T$, is a flow on $(S, \mathcal{B}, \mu)$ if

(i) $\phi_{t_{1}+t_{2}}(s)=\phi_{t_{2}}\left(\phi_{t_{1}}(s)\right)$ for all $t_{1}, t_{2} \in T, s \in S$;

(ii) $\phi_{0}(s)=s$ for all $s \in S$. 
A flow $\phi$ is said to be measurable if $\phi_{t}(s)$ is a measurable map from $T \times S$ to $S$. A flow $\phi$ is said to be nonsingular if $\mu\left(\phi_{t}^{-1}(A)\right)=0$ is equivalent to $\mu(A)=0$ for all $A \in \mathscr{B}, t \in T$.

The next result relates the spectral functions of stationary $\alpha$-Fréchet processes to flows.

Theorem 5.1. Let $\left\{X_{t}\right\}_{t \in T}$ be a stationary $\alpha$-Fréchet process with a measurable minimal representation $\left\{f_{t}\right\}_{t \in T} \subset L_{+}^{\alpha}(S, \mu)$. Then, there exists a unique, modulo $\mu$, nonsingular, and measurable flow $\left\{\phi_{t}\right\}_{t \in T}$ such that

$$
f_{t}(s)=\left(\frac{d\left(\mu \circ \phi_{t}\right)}{d \mu}\right)^{1 / \alpha}(s)\left(f_{0} \circ \phi_{t}\right)(s) \quad \mu \text {-a.e. for all } t \in T \text {. }
$$

Theorem 5.1 is stronger than Theorem 6.1 of [9], where the measurability is not considered and the flow structure is not explicitly explored. The proof is given in [35]. For the readers familiar with Rosiński's work [26], this result is similar to Theorem 3.1 therein. In view of this result, we will say that a stationary $\alpha$-Fréchet measurable process $\left\{X_{t}\right\}_{t \in T}$ is generated by the nonsingular measurable flow $\left\{\phi_{t}\right\}_{t \in T}$ on $(S, \mu)$ if it has a spectral representation $\left\{f_{t}\right\}_{t \in T} \subset L_{+}^{\alpha}(S, \mu)$ as in (5.1) and

$$
\operatorname{supp}\left\{f_{0} \circ \phi_{t}: t \in T\right\}=S \quad \mu \text {-a.e. }
$$

Here the spectral representation $\left\{f_{t}\right\}_{t \in T} \subset L_{+}^{\alpha}(S, \mu)$ is not necessarily minimal, but it is assumed to have full support in the sense that (5.2) holds. The minimality condition plays a crucial role in the proof of the existence of flow representations in Theorem 5.1.

Not all flow representations are minimal. We can show (see Proposition 6.1 of [35]), however, that any two flows corresponding to minimal representations of the same $\alpha$-Fréchet process are equivalent. We say that two measurable, nonsingular flows $\left\{\phi_{t}^{(i)}\right\}_{t \in T}$ on $\left(S_{i}, \mu_{i}\right), i=1,2$, are equivalent, written $\left\{\phi_{t}^{(1)}\right\}_{t \in T} \sim^{\Phi}\left\{\phi_{t}^{(2)}\right\}_{t \in T}$, if there exists a measurable map $\Phi: S_{2} \rightarrow S_{1}$ such that (i) there exist $N_{i} \subset S_{i}$ with $\mu_{i}\left(N_{i}\right)=0, i=1,2$, such that $\Phi$ is a Borel isomorphism between $S_{2} \backslash N_{2}$ and $S_{1} \backslash N_{1}$; (ii) $\mu_{1}$ and $\mu_{2} \circ \Phi^{-1}$ are mutually absolutely continuous; and (iii) $\phi_{t}^{(1)} \circ \Phi=\Phi \circ \phi_{t}^{(2)}, \mu_{2}$-a.e. for each $t \in T$.

\subsection{Decompositions induced by nonsingular flows}

The decompositions introduced in Examples 4.3 and 4.4 are motivated by corresponding notions from ergodic theory. Consider a measure space $(S, \mu)$ and a measurable, nonsingular $\operatorname{map} \phi: S \rightarrow S$. A measurable set $B \subset S$ is said to be (i) wandering if $\phi^{-n}(B), n=0,1,2, \ldots$, are disjoint; and (ii) weakly wandering if $\phi^{-n_{k}}(B), n_{k} \in \mathbb{N}$, are disjoint for some infinite sequence $0=n_{0}<n_{1}<\cdots$.

Now we give two decompositions for max-stable processes. Their counterparts for sumstable processes have been thoroughly studied (see [26] and [30]).

Hopf (conservative-dissipative) decomposition. The map $\phi$ is said to be conservative if there is no wandering measurable set $B \subset S$, with positive measure $\mu(B)>0$. We can show that, for any measurable, nonsingular map $\phi: S \rightarrow S$, there exists a partition of $S$ into two disjoint measurable sets $S=C \cup D, C \cap D=\varnothing$, such that (i) $C$ and $D$ are $\phi$-invariant; (ii) $\phi: C \rightarrow C$ is conservative and $D=\bigcup_{k \in \mathbb{Z}} \phi^{k}(B)$ for some wandering set $B \subset S$. This decomposition is unique (modulo $\mu$ ) and is called the Hopf decomposition of $S$ with respect to $\phi$. If the component $C$ is trivial, i.e. $\mu(C)=0$, then $\phi$ is said to be dissipative. The restrictions $\phi: C \rightarrow C$ and $\phi: D \rightarrow D$ are the conservative and dissipative components of the mapping $\phi$, respectively. 
Now, given a jointly measurable, nonsingular flow $(t, s) \mapsto \phi_{t}(s), t \in T, s \in S$, we can consider the Hopf decompositions $S=C_{t} \cup D_{t}$ for each $\phi_{t}, t \in T \backslash\{0\}$. By the measurability, however, it follows that $\mu\left(C_{t} \Delta C\right)=\mu\left(D_{t} \Delta D\right)=0$ for some $C \cap D=\varnothing, S=C \cup D$ (see, e.g. [18] and [26]). We thus find that any measurable, nonsingular flow $\left\{\phi_{t}\right\}_{t \in T}$ has a Hopf decomposition $S=C \cup D$, where $\phi^{C}:=\left\{\left.\phi_{t}\right|_{C}\right\}_{t \in T}$ and $\phi^{D}:=\left\{\left.\phi_{t}\right|_{D}\right\}_{t \in T}$ are conservative and dissipative flows, respectively.

The following result is an immediate consequence from the proofs of Theorem 4.1 and Corollary 4.2 of [26].

Theorem 5.2. Let $X=\left\{X_{t}\right\}_{t \in T}$ be a stationary $\alpha$-Fréchet process with measurable representation $\left\{f_{t}(s)\right\}_{t \in T} \subset L_{+}^{\alpha}(S, \mu)$ of full support. Then

(i) $X$ is generated by a conservative flow if and only if

$$
\int_{T} f_{t}^{\alpha}(s) \lambda(\mathrm{d} t)=\infty \text { for } \mu \text {-almost all } s \in S ;
$$

(ii) $X$ is generated by a dissipative flow if and only if

$$
\int_{T} f_{t}^{\alpha}(s) \lambda(\mathrm{d} t)<\infty \text { for } \mu \text {-almost all } s \in S
$$

(iii) if $X$ is generated by a conservative or dissipative flow in one measurable representation, then in any other measurable representation of $X$, it is still generated by a conservative or, respectively, dissipative flow.

This result justifies the terminology in the conservative-dissipative decomposition of Example 4.3. In particular, the sets $C$ and $D$ in (4.11) correspond precisely to the conservative and dissipative parts in the Hopf decomposition of the flow $\left\{\phi_{t}\right\}_{t \in T}$ associated with the process $X$.

Positive-null decomposition. If we replace 'wandering' by 'weakly wandering' in the Hopf decomposition, we obtain the so-called positive-null decomposition of $S$. Alternatively, the map $\phi$ is said to be positive if there exists a finite measure $v \sim \mu$ such that $\phi$ is $v$-invariant. In this case, there are no weakly wandering sets $B$ of positive $\mu$-measure (or, equivalently, $\nu$-measure). For any nonsingular map $\phi$, there exists a partition $S=P \cup N$, unique modulo $\mu$, such that $P$ and $N$ are disjoint, measurable, and $\phi$-invariant. Furthermore, $\phi: P \rightarrow P$ is positive, and $N=\bigcup_{k \geq 0} \phi^{-n_{k}}(B)$ for some disjoint $\phi^{-n_{k}}(B)$ s, where $B$ is weakly wandering. The sets $N$ and $P$ are called the null-recurrent and, respectively, positive-recurrent parts of $S$, with respect to the map $\phi$ (see, e.g. Section 1.4 of [1]).

As in the case of the Hopf decomposition, a jointly measurable, nonsingular flow $\left\{\phi_{t}\right\}_{t \in T}$ gives rise to a positive-null decomposition: $S=P \cup N$, where $\mu\left(P_{t} \Delta P\right)=\mu\left(N_{t} \Delta N\right)=0$ for all $t \in T \backslash\{0\}$, and where $S=P_{t} \cup N_{t}$ is the positive-null decomposition of the map $\phi_{t}, t \in T \backslash\{0\}$ (see, e.g. [18] and [30]).

Theorem 2.1 of Samorodnitsky [30] about symmetric $\alpha$-stable processes applies mutatis mutandis to the max-stable case.

Theorem 5.3. Let $X=\left\{X_{t}\right\}_{t \in T}$ be a stationary $\alpha$-Fréchet process with measurable representation $\left\{f_{t}(s)\right\}_{t \in T} \subset L_{+}^{\alpha}(S, \mu)$ of full support. Then

(i) $X$ is generated by a positive flow if and only if, for all $w \in \mathcal{W}$,

$$
\int_{T} w(t) f_{t}^{\alpha}(s) \lambda(\mathrm{d} t)=\infty \text { for } \mu \text {-almost all } s \in S,
$$

where $\mathcal{W}$ is as in (4.12); 
(ii) $X$ is generated by a null flow if and only if, for some $w \in \mathcal{W}$,

$$
\int_{T} w(t) f_{t}^{\alpha}(s) \lambda(\mathrm{d} t)<\infty \text { for } \mu \text {-almost all } s \in S ;
$$

(iii) if $X$ is generated by a positive or null flow in one measurable representation, then in any other measurable representation of $X$, it is still generated by a positive or, respectively, null flow.

As in the Hopf decomposition, Theorem 5.3 shows that the components $X^{\text {pos }}$ and $X^{\text {null }}$ in decomposition (4.13) are generated by positive- and null-recurrent flows, respectively. This is because the sets $P$ and $N$ in (4.15) yield the positive-null decomposition of a flow $\left\{\phi_{t}\right\}_{t \in T}$ associated with $X$.

\subsection{Examples}

Here, we collect some structural results and observations on the interplay between the three types of classification of max-stable processes discussed above. Namely, (i) continuousdiscrete; (ii) conservative-dissipative; and (iii) positive-null.

Theorems 5.2 and 5.3 imply that the positive component of a max-stable process is conservative and the dissipative component is null-recurrent. Thus, for a measurable stationary $\alpha$-Fréchet process $\left\{X_{t}\right\}_{t \in T}$, we have the decomposition

$$
\left\{X_{t}\right\}_{t \in T} \stackrel{\mathrm{D}}{=}\left\{X_{t}^{\text {pos }} \vee X_{t}^{C, \text { null }} \vee X_{t}^{D}\right\}_{t \in T},
$$

where $X_{t}^{C}=X_{t}^{\mathrm{pos}} \vee X_{t}^{C \text {,null }}$ and $X_{t}^{\text {null }}=X_{t}^{C \text {,null }} \vee X_{t}^{D}, t \in T$. Here $X^{\text {pos }}, X^{C \text {, null }}$, and $X^{D}$ are independent $\alpha$-Fréchet processes; $X^{\text {pos }}$ is positive-recurrent and conservative, $X^{D}$ is dissipative and null-recurrent, and $X^{C \text {,null }}$ is conservative and null-recurrent. We will see that $X^{D}$ is precisely the mixed moving maxima. Moreover, we show that the spectrally discrete component has no conservative-null component $X^{C \text {,null }}$.

The following theorem shows that the purely dissipative stationary $\alpha$-Fréchet processes are precisely the mixed moving maxima.

Theorem 5.4. Let $\left\{X_{t}\right\}_{t \in T}$ be a measurable stationary $\alpha$-Fréchet process. This process is generated by a dissipative flow if and only if there exist a Borel space $W$, a $\sigma$-finite measure $v$ on $W$, and a function $g \in L_{+}^{\alpha}(W \times T, v \otimes \lambda)$ such that

$$
\left\{X_{t}\right\}_{t \in T} \stackrel{\mathrm{D}}{=}\left\{\int_{W \times T}^{\mathrm{e}} g(x, t+u) M_{\alpha}(\mathrm{d} x, \mathrm{~d} u)\right\}_{t \in T} .
$$

Here $M_{\alpha}$ is an $\alpha$-Fréchet random sup-measure on $W \times T$ with the control measure $\nu \otimes \lambda$, and $\lambda$ is the Lebesgue measure if $T=\mathbb{R}$ and the counting measure if $T=\mathbb{Z}$. Moreover, we can always choose $(W, v)$ and $g$ such that the representation $g_{t}(x, u):=g(x, t+u)$ is minimal.

Proof. Since $g \in L_{+}^{\alpha}(W \times T, v \otimes \lambda)$, Fubini's theorem implies that, for almost all $(x, u) \in$ $W \times T, \int_{T} g(x, t+u)^{\alpha} \lambda(\mathrm{d} t)<\infty$. This, in view of (4.10) implies that $X$ is dissipative.

The 'only if' part follows as in the proof of Theorem 4.4 of [26] from the results of [17].

Remark 5.1. Theorem 5.4 parallels the fact that the class of stationary and dissipative symmetric $\alpha$-stable processes is precisely the class of mixed moving averages (see Theorem 4.4 of [26]). Recently, Kabluchko [14] established the same result as in Theorem 5.4 using an interesting association device between $\alpha$-Fréchet $(\alpha \in(0,2))$ and symmetric $\alpha$-stable processes. 
As shown in [33], the mixed moving maxima processes are mixing and, hence, ergodic. See also the recent work of Kabluchko and Schlather [15]. Thus, Theorem 5.4 implies that the dissipative component of a max-stable process is mixing. On the other hand, Samorodnitsky [30, Theorem 3.1] showed that stationary symmetric $\alpha$-stable processes are ergodic if and only if they are generated by a null-recurrent flow. Kabluchko [14, Theorem 8] showed that this continues to be the case for stationary $\alpha$-Fréchet processes.

The previous discussion shows that the ergodic and mixing properties of the null and dissipative components are in line with the decomposition $X_{t}^{\text {null }}=X_{t}^{D} \vee X_{t}^{C \text {, null }}, t \in T$. An example of conservative-null flow can be found in [30]. This yields nontrivial examples of sum- and max-stable processes that are conservative and null. We are not aware, however, of an example of an ergodic max-stable process that is not mixing.

The next two results clarify the structure of the stationary spectrally discrete processes in discrete $(T=\mathbb{Z})$ and continuous $(T=\mathbb{R})$ time, respectively. We first show that, for spectrally discrete stationary max-stable time series, the conservative-dissipative and positivenull decompositions coincide. That is, such processes have no conservative-null components. Moreover, the dissipative (equivalently, null-recurrent) component does not exist if the time series has only a finite number of principal components. Recall the definition of the spectrally discrete component $X^{N}=\left\{X_{t}^{N}\right\}_{t \in T}$ in (4.1).

Proposition 5.1. Let $X=\left\{X_{t}\right\}_{t \in T}$, with $T=\mathbb{Z}$, be a stationary $\alpha$-Fréchet process (time series).

(i) $X^{N}$ has no conservative-null component, i.e. $X^{N, C, \text { null }}=0$.

(ii) If $1 \leq N<\infty$ then $X^{N}$ is necessarily conservative, and, equivalently, positive recurrent.

Proof. Without loss of generality, suppose that $X=X^{N}$ and $\left\{f_{t}(s)\right\}_{t \in T} \subset L_{+}^{\alpha}\left(S^{N}, \lambda_{N}\right)$ is a minimal representation with standardized support for $X$. We have

$$
f_{t}=\left(\frac{d\left(\lambda_{N} \circ \phi_{t}\right)}{d \lambda_{N}}\right)^{1 / \alpha} f_{0} \circ \phi_{t} \quad \text { for all } t \in T,
$$

where $\phi_{t}: S^{N} \rightarrow S^{N}$ is a nonsingular flow on $\left(S^{N}, \lambda_{N}\right)$. Since $S^{N} \subset \mathbb{N}$ and $\lambda_{N}$ is the counting measure, the nonsingular transformations are necessarily measure preserving, i.e. permutations. Thus, the term $\mathrm{d}\left(\lambda_{N} \circ \phi_{t}\right) / \mathrm{d} \lambda_{N} \equiv 1$ and $f_{t}(s)=f_{0} \circ \phi_{t}(s)$.

We start by proving (ii). Since $\phi_{1}:\{1, \ldots, N\} \rightarrow\{1, \ldots, N\}$ is a permutation, it has a finite invariant measure and, hence, the flow $\left\{\phi_{t}\right\}_{t \in T}$ is positive-recurrent and hence conservative.

Now we prove (i). Note that when $1 \leq N<\infty$, we have shown in (ii) that $X^{N}$ is conservative and positive-recurrent. For $N=\infty$, we consider two cases. First we suppose that, for every $s \in S^{N}$, the recurrent time

$$
\tau_{s}:=\inf \left\{t>0: \phi_{t}(s)=s\right\}
$$

is finite. Let $\mathfrak{O}(s)$ denote the orbit of state $s$ with respect to flow $\left\{\phi_{t}\right\}_{t \in T}$, i.e. $\mathfrak{O}(s):=\left\{\phi_{t}(s)\right.$ : $t \in T\}$. Every orbit of $\left\{\phi_{t}\right\}_{t \in T}$ is $\tau_{s}$-periodic, i.e. $|\mathfrak{O}(s)|<\infty$. Since $N=\infty$, the total number of different orbits must be infinite. Enumerate all the orbits by $\mathfrak{O}_{1}, \mathfrak{O}_{2}, \ldots$, so that $\mathfrak{O}(s)=\mathfrak{O}_{\pi(s)}$ with $\pi: S^{N} \rightarrow \mathbb{N}$ and $S^{N}=\bigcup_{k \in \mathbb{N}} \mathfrak{O}_{k}$. Observe that the orbits are disjoint. We now define a finite invariant measure on $S^{N}$, equivalent to the counting measure:

$$
\tilde{\lambda}(\{s\}):=2^{-\pi(s)} \frac{1}{\left|\mathfrak{O}_{\pi(s)}\right|} \quad \text { for all } s \in S^{N} .
$$


This measure is clearly invariant on each $\mathfrak{O}_{k}$ for all $k \in \mathbb{N}$. Since $\tilde{\lambda}\left(\mathfrak{O}_{k}\right)=2^{-k}$, the measure $\tilde{\lambda}$ is finite and it is clearly equivalent to the counting measure. Thus, $X^{N}$ is positive and conservative.

On the other hand, suppose that there exists a state $s$ with $\tau_{s}=\infty$. Then, its orbit is infinite and nonrecurrent, i.e. $\left|\mathfrak{O}_{k}(s)\right|=\infty$. Then, the flow $\left\{\phi_{t}\right\}_{t \in T}$ is both null-recurrent and dissipative on $\mathfrak{O}_{k}(s)$. Indeed, the null recurrence follows from the fact that there is no positive finite invariant measure on $\mathfrak{O}_{k}(s)$. The dissipativity follows from the remark that $\mathfrak{O}_{k}(s)=\bigcup_{j \in \mathbb{Z}} \phi_{j}(s)$ is a disjoint union. We have thus shown that $\left\{\phi_{t}\right\}_{t \in T}$ is dissipative and null-recurrent on nonrecurrent orbits.

The following result shows that the continuous-time stationary, measurable, and spectrally discrete max-stable processes are trivial.

Theorem 5.5. Let $X=\left\{X_{t}\right\}_{t \in T}$, with $T=\mathbb{R}$, be a stationary and measurable $\alpha$-Fréchet process. If $N \geq 1$ then it must be $N=1$. That is, the spectrally discrete component $X^{N}$ is the random constant process: $\left\{X_{t}^{N}\right\}_{t \in \mathbb{R}} \stackrel{\mathrm{D}}{=}\{Z\}_{t \in \mathbb{R}}$ for some $\alpha$-Fréchet variable $Z$.

Proof. Let $\left\{f_{t}\right\}_{t \in T}$ and $\left\{\phi_{t}\right\}_{t \in T}$ be as in Proposition 5.1. Observe moreover that in this case the $\phi_{t}$ s are measure-preserving bijections, and in view of Theorem 5.1, the flow $\left\{\phi_{t}(s)\right\}$ is measurable. For any fixed $s \in S^{N}$, consider $\tau_{s}$ defined in (5.3). The proof consists of three steps.

Step 1: show that $\tau_{s}=0$ implies that $\phi_{t}(s) \equiv s$ for all $t \in \mathbb{R}$. Indeed, suppose that $\tau_{s}=0$ and note that, by definition, for all $n>0$, there exist $0<t_{n, 1}<t_{n, 2}<1 / n$ such that $\phi_{t_{n, 1}}(s)=\phi_{t_{n, 2}}(s)=s$. Set $T_{0}:=\bigcup_{n \in \mathbb{N}} \bigcup_{k \in \mathbb{Z}}\left\{t_{n, 1}+k\left(t_{n, 2}-t_{n, 1}\right)\right\}$. It follows that $T_{0}$ is dense in $\mathbb{R}$ and $\phi_{t}(s)=s$ for all $t \in T_{0}$. Hence, $f_{t}(s)=f_{0} \circ \phi_{t}(s)=f_{0}(s)$ for all $t \in T_{0}$. Now, we define a new $\alpha$-Fréchet process $Y=\left\{Y_{t}\right\}_{t \in T}$ :

$$
Y_{t}:=\int_{S^{N}}^{\mathrm{e}} \mathbf{1}_{\left\{\phi_{t}(r)=s\right\}} M_{\alpha}(\mathrm{d} r) \quad \text { for all } t \in T .
$$

Since $\left\{\phi_{t}\right\}_{t \in T}$ is a flow, $\phi_{t}$ is invertible for any $t \in T_{0}$. Hence, for all $t \in T_{0}$, we have $\phi_{t}(r)=\phi_{t}(s) \equiv s$ if and only if $r=s$. This shows that, for all $t \in T_{0}$,

$$
\mathbf{1}_{\left\{\phi_{t}(r)=s\right\}}=\mathbf{1}_{\left\{\phi_{0}(r)=s\right\}}=\mathbf{1}_{\{r=s\}},
$$

which implies that $Y_{t}=Y_{0}$ almost surely (a.s.) for all $t \in T_{0}$. Moreover, as $\left\{\phi_{t}\right\}_{t \in T}$ is measurable, so is $\left\{Y_{t}\right\}_{t \in T}$ by Proposition 4.1. Also, $Y=\left\{Y_{t}\right\}_{t \in T}$ is stationary, since it is generated by a measure-preserving flow. Thus, the stationarity and measurability of $Y$ imply that it is continuous in probability (see Theorem 3.1 of [33]). This, and the fact that $Y_{t}=Y_{0}$ a.s. for all $t$ in a dense subset $T_{0}$ of $\mathbb{R}$, imply that $Y_{t}=Y_{0}$ a.s. for all $t \in \mathbb{R}$. Therefore, by (5.4), for the spectral functions, we obtain $\mathbf{1}_{\left\{\phi_{t}(r)=s\right\}}=\mathbf{1}_{\{r=s\}}$ for all $r \in S^{N}, t \in \mathbb{R}$. This shows that $\phi_{t}(s)=s$ for all $t \in \mathbb{R}$.

Step 2: show that $\tau_{s}>0$ implies that $\phi_{\tau_{s}}(s)=s$. Suppose that $\phi_{\tau_{s}}(s) \neq s$. Then, as above, there exist $t_{1}, t_{2} \in\left(\tau_{s}, \tau_{s}+\tau_{s} / 2\right)$ such that $\phi_{t_{1}}(s)=\phi_{t_{2}}(s)=s$. But, it follows that $\phi_{t_{1}+k\left(t_{2}-t_{1}\right)}(s)=s$ for all $k \in \mathbb{Z}$. This, since $\left\{t_{1}+k\left(t_{2}-t_{1}\right)\right\}_{k \in \mathbb{Z}} \cap\left(0, \tau_{s}\right) \neq \varnothing$, contradicts the definition of $\tau_{s}$.

Step 3: show that it is impossible to have $\tau_{s}>0$ for all $s \in S^{N}$. Write $\mathfrak{T}_{s}=\left\{t: \phi_{t}\left(s_{0}\right)=\right.$ $s$ for some $s_{0} \in S^{N}$. Observe that the set $\mathfrak{T}_{s}$ is countably infinite for all $s \in S^{N}$ such that $\tau_{s}>0$, since by step 2 above, $\mathfrak{T}_{s}=\left\{k \tau_{s}\right\}_{k \in \mathbb{Z}}$. Note also that $\bigcup_{s \in S^{N}} \mathfrak{T}_{s}=\mathbb{R}$. However, the assumption that $\tau_{s}>0$ for all $s \in S^{N}$ would imply that $\bigcup_{s \in S^{N}} \mathfrak{T}_{s}$ has the cardinality of $\mathbb{N}$, which is a contradiction. 
We now conclude the proof. By step 3 above, there must exist $s \in S^{N}$ such that $\tau_{s}=0$. Set $\mathfrak{R}=\left\{s \in S^{N}: \phi_{t}(s)=s\right.$ for all $\left.t \in \mathbb{R}\right\}$. We have already seen in step 1 that $\tau_{s}=0$ implies that $\phi_{t}(s) \equiv s$ for all $t \in \mathbb{R}$, whence $\Re$ is $\phi$-invariant. Consider now a new $\alpha$-Fréchet process:

$$
\left\{Y_{t}\right\}_{t \in T} \stackrel{\mathrm{D}}{=}\left\{\int_{S^{N} \backslash \mathfrak{R}}^{\mathrm{e}} f_{t}(r) M_{\alpha}(\mathrm{d} r)\right\}_{t \in T} .
$$

Since $S^{N} \backslash \mathfrak{R}$ is $\phi$-invariant, the last representation is also minimal and has standardized support. Moreover, the process $Y$ is generated by the flow $\left\{\phi_{t}\right\}_{t \in \mathbb{R}}$, restricted to $S^{N} \backslash \mathfrak{R}$. Since $\tau_{s}>0$ for all $s \in S^{N} \backslash \mathfrak{R}$, by step 3 , it follows that $S^{N} \backslash \mathfrak{R}=\varnothing$.

On the other hand, since $\phi_{t}(s) \equiv s$ for all $t \in \mathbb{R}, s \in \mathfrak{R}$, the minimality of $\left\{f_{t}\right\}_{t \in T}$ implies that $|\mathfrak{R}|=\left|S^{N}\right|=1$. Therefore, $\left\{X_{t}\right\}_{t \in T} \stackrel{\mathrm{D}}{=}\{Z\}_{t \in T}$ for some $\alpha$-Fréchet random variable $Z$.

Example 5.1. In contrast with Proposition 5.1(i), the spectrally discrete component of a stationary $\alpha$-Fréchet time series may be dissipative if it involves an infinite number of principal components. Indeed, by Theorem 5.4, the moving maxima $X_{t}:={ }^{\mathrm{e}} \int_{\mathbb{Z}} f(t+s) M_{\alpha}(\mathrm{d} s) \equiv$ $\bigvee_{i \in \mathbb{Z}} f(t+i) M_{\alpha}(\{i\})$ is dissipative and spectrally discrete, where $M_{\alpha}$ has the counting control measure on $\mathbb{Z}$.

\section{Brown-Resnick processes}

Consider the following doubly stochastic process (see, e.g. [16] and [33]):

$$
\left\{X_{t}\right\}_{t \in \mathbb{R}} \stackrel{\mathrm{D}}{=}\left\{\int_{E}^{\mathrm{e}} \mathrm{e}^{W_{t}-\sigma_{t}^{2} / 2} \mathrm{~d} M_{1}\right\}_{t \in \mathbb{R}} .
$$

Here $W_{t}$ is a zero-mean Gaussian process defined on the probability space $(E, \mathcal{E}, \mu)$ with variance $\sigma_{t}^{2}$, and $M_{1}$ is a 1-Fréchet random sup-measure on $E$ with control measure $\mu$. Since $\mathrm{E}_{\mu} \mathrm{e}^{W_{t}-\sigma_{t}^{2} / 2}=1<\infty$, the 1-Fréchet process in (6.1) is well defined. The processes having representation (6.1) were first introduced by Brown and Resnick [4] with $W_{t}$ being the standard Brownian motion. In general, we will call $\left\{X_{t}\right\}_{t \in \mathbb{R}}$ as in (6.1) a Brown-Resnick 1-Fréchet process.

Kabluchko et al. [16] showed that if $\left\{W_{t}\right\}_{t \in \mathbb{R}}$ has stationary increments then the BrownResnick process $\left\{X_{t}\right\}_{t \in \mathbb{R}}$ in (6.1) is stationary. The following interesting result about an arbitrary zero-mean Gaussian process with stationary increments and continuous paths is obtained by combining the results of [16] and our Theorems 5.2 and 5.4.

Theorem 6.1. Let $W=\left\{W_{t}\right\}_{t \in \mathbb{R}}$ be a Gaussian zero-mean process with stationary increments and continuous paths. If

$$
\lim _{|t| \rightarrow \infty}\left(W_{t}-\frac{1}{2} \sigma_{t}^{2}\right)=-\infty \text { a.s. }
$$

then

$$
\int_{-\infty}^{\infty} \mathrm{e}^{W_{t}-\sigma_{t}^{2} / 2} \mathrm{~d} t<\infty \quad \text { a.s. }
$$

where $\sigma_{t}^{2}=\mathrm{E} W_{t}^{2}=\operatorname{var}\left(W_{t}\right)$.

Proof. Let $\left\{X_{t}\right\}_{t \in \mathbb{R}}$ be the Brown-Resnick process defined in (6.1). The process $\left\{\log X_{t}\right\}_{t \in \mathbb{R}}$ is also max-stable but it has Gumbel marginals. Kabluchko et al. [16] showed that $\left\{\log X_{t}\right\}_{t \in \mathbb{R}}$ is stationary and, hence, so is $\left\{X_{t}\right\}_{t \in \mathbb{R}}$. Moreover, by Theorem 13 of [16], condition (6.2) implies that $\left\{\log X_{t}\right\}_{t \in \mathbb{R}}$, or, equivalently, $\left\{X_{t}\right\}_{t \in \mathbb{R}}$ has a mixed moving maxima representation. On the 
other hand, Theorem 5.4 implies that any process with mixed moving maxima representation is dissipative. Dissipativity of $\left\{X_{t}\right\}_{t \in \mathbb{R}}$ is equivalent to (6.3) by Theorem 5.2. This completes the proof.

The following question arises.

Question 6.1. For what general classes of continuous-path, zero-mean Gaussian processes $\left\{W_{t}\right\}_{t \in \mathbb{R}}$ with stationary increments is the Brown-Resnick stationary process (6.1) dissipative?

The next result provides a partial answer to this question for the interesting case when $W=\left\{W_{t}\right\}_{t \in \mathbb{R}}$ is the fractional Brownian motion (FBM). Recall that the FBM is a zeromean Gaussian process with stationary increments, which is self-similar. The process $W$ is said to be self-similar with self-similarity parameter $H>0$ if, for all $c>0$, we have $\left\{W_{c t}\right\}_{t \in \mathbb{R}} \stackrel{\mathrm{D}}{=}\left\{c^{H} W_{t}\right\}_{t \in \mathbb{R}}$. The FBM necessarily has the covariance function

$$
\mathrm{E} W_{t} W_{s}=\frac{\sigma^{2}}{2}\left(|t|^{2 H}+|s|^{2 H}-|t-s|^{2 H}\right) \quad \text { with } t, s \in \mathbb{R},
$$

where $0<H \leq 1$ is the self-similarity parameter of $W$. The fractional Brownian motions have versions with continuous paths (see, e.g. [31, p. 490]).

Proposition 6.1. The stationary Brown-Resnick processes $X=\left\{X_{t}\right\}_{t \in \mathbb{R}}$ associated with the FBMs $\left\{W_{t}\right\}_{t \in \mathbb{R}}$ in (6.4) are dissipative and, hence, they have mixed moving maxima representations.

Proof. Without loss of generality, we suppose that the FBM $W$ has continuous paths. As indicated above, the stationarity of $X$ follows from the fact that $W$ has stationary increments (see [16]). Now, by Theorem 5.2, $X$ is dissipative if and only if (6.3) holds. By Theorem 6.1, it suffices to show that (6.2) holds.

By the law of the iterated logarithm for Gaussian processes (see [19]), we have

$$
\limsup _{t \rightarrow \infty} \frac{W_{t}}{\sqrt{2 \sigma_{t}^{2} \log \log t}}=1 \quad \text { a.s. }
$$

with $\sigma_{t}^{2}=\sigma^{2}|t|^{2 H}$. It is clear that (6.2) follows from (6.5). The proof is thus complete.

Observe that the above result continues to hold even in the degenerate case $H=1$. We then have $W_{t}=t Z, t \in \mathbb{R}$, where $Z$ is a zero-mean Gaussian random variable. In this case, the corresponding Brown-Resnick process has a simple moving maxima representation. Indeed, for simplicity, let $\sigma^{2}=\operatorname{var}(Z)=1$ and observe that

$$
X_{t}:=\int_{E}^{\mathrm{e}} \mathrm{e}^{t Z(u)-t^{2} / 2} M_{1}(\mathrm{~d} u)=\int_{E}^{\mathrm{e}} \mathrm{e}^{Z^{2}(u) / 2} \mathrm{e}^{-(t-Z(u))^{2} / 2} M_{1}(\mathrm{~d} u) .
$$

Note that the measure $v(A):=\int_{E} \mathbf{1}_{\{Z(u) \in A\}} \mathrm{e}^{Z^{2}(u) / 2} \mu(\mathrm{d} u) \equiv \lambda(A) / \sqrt{2 \pi}$ is up to a constant factor equal to the Lebesgue measure $\lambda$ on $\mathbb{R}$. Therefore, we can show that

$$
\left\{X_{t}\right\}_{t \in \mathbb{R}} \stackrel{\mathrm{D}}{=}\left\{\frac{1}{\sqrt{2 \pi}} \int_{\mathbb{R}}^{\mathrm{e}} \mathrm{e}^{-(t-z)^{2} / 2} \tilde{M}_{1}(\mathrm{~d} z)\right\}_{t \in \mathbb{R}},
$$

where $\widetilde{M}_{1}$ is a 1-Fréchet random sup-measure with the Lebesgue control measure. This shows that $X$ in this simple case is merely a moving maxima rather than a mixed moving maxima. 
We have thus shown that the Brown-Resnick process (6.1) driven by FBM $\left\{W_{t}\right\}_{t \in T}$ is purely dissipative. Thus, by Theorem 5.4, $\left\{X_{t}\right\}_{t \in T}$ is a mixed moving maxima. In two very recent papers [14], [16], Kabluchko and co-authors established very similar classification results by using very different methods based on Poisson point processes on abstract path spaces. Their approach directly yielded the moving maxima representation (and, hence, dissipativity) of the Brown-Resnick-type processes $X$ under the alternative condition (6.2). This condition is only shown to be sufficient for dissipativity of $X$. Its relationship with our necessary and sufficient condition (6.3) is a question of independent interest.

The question raised in Kabluchko [14] on whether there exist stationary Brown-Resnick processes $X$ of mixed type, i.e. with nontrivial dissipative and conservative components, still remains open. In view of our new necessary and sufficient condition (6.3), this question is equivalent to the following.

Question 6.2. Is it true that $\mu\left\{\int_{-\infty}^{\infty} \mathrm{e}^{W_{t}-\sigma_{t}^{2} / 2} \mathrm{~d} t<\infty\right\} \in\{0,1\}$ for Gaussian processes $W=$ $\left\{W_{t}\right\}_{t \in \mathbb{R}}$ with stationary increments and continuous paths?

\section{Appendix A. Proofs}

Proof of Proposition 4.1. To prove part (i), observe that, since $\mu$ is $\sigma$-finite, it is enough to focus on the case when $\mu$ is a probability measure: $\mu(S)=1$. Thus, $\left\{f_{t}(s)\right\}_{t \in T}$ may be viewed as a stochastic process, defined on the probability space $\left(S, \mathscr{B}_{S}, \mu\right)$.

Note that $L_{+}^{\alpha}(S, \mu)$ equipped with the metric $\rho_{\mu, \alpha}(f, g)=\int_{S}\left|f^{\alpha}-g^{\alpha}\right| \mathrm{d} \mu$ is a complete separable metric space. Furthermore, $\rho_{\mu, \alpha}$ metrizes the convergence in probability in the space $(S, \mu)$. Therefore, Theorem 3 of [5] (see also Proposition 9.4.4 of [31]) implies that the stochastic process $f=\left\{f_{t}(s)\right\}_{t \in T}$ has a measurable modification if and only if the map $h_{f}: t \mapsto\left[f_{t}\right]$ is Borel-measurable and has separable range $h_{f}(T)$. Here $[f]$ denotes the class of all $L_{+}^{\alpha}(S, \mu)$-functions, equal to $f$, $\mu$-a.e.

Similarly, $X=\left\{X_{t}\right\}_{t \in T}$ has a measurable modification if and only if $h_{X}: t \mapsto\left[X_{t}\right]$ is Borel-measurable and has separable range $h_{X}(T)$, where $\left[X_{t}\right] \in \mathcal{L}^{0}(\Omega, \mathcal{A}, \mathrm{P})$ is equipped with a metric, which metrizes the convergence in probability. Here $\mathcal{L}^{0}(\Omega, \mathcal{A}, \mathrm{P})$ denotes the collection of equivalence classes of random variables, with respect to the relation of almost-sure equality. We focus on the set $\mathcal{M}=\left\{[\xi]: \xi={ }^{\mathrm{e}} \int_{S} g \mathrm{~d} M_{\alpha}, g \in L_{+}^{\alpha}(S, \mu)\right\}$, which is a closed subset of $\mathcal{L}^{0}(\Omega, \mathcal{A}, \mathrm{P})$ with respect to the convergence in probability. Theorem 2.1 of [34] shows that since $\left(L_{+}^{\alpha}(S, \mu), \rho\right)$ is complete and separable, so is $\mathcal{M}$ with respect to the metric

$$
\rho_{\mathcal{M}}(\xi, \eta):=2\|\xi \vee \eta\|_{\alpha}^{\alpha}-\|\xi\|_{\alpha}^{\alpha}-\|\eta\|_{\alpha}^{\alpha} .
$$

Furthermore, $\rho_{\mathcal{M}}$ metrizes the convergence in probability and we have

$$
\rho_{\mathcal{M}}(\xi, \eta)=\int_{S}\left|f^{\alpha}-g^{\alpha}\right| \mathrm{d} \mu \equiv \rho_{\mu, \alpha}(f, g)
$$

for all $\xi=\int_{S}^{\mathrm{e}} f \mathrm{~d} M_{\alpha}$ and $\eta={ }^{\mathrm{e}} \int_{S} g \mathrm{~d} M_{\alpha}$, with $f, g \in L_{+}^{\alpha}(S, \mu)$.

Now, the separability of $L_{+}^{\alpha}(S, \mu)$ and $\mathcal{M}$ implies the separability of the ranges $h_{f}(T) \subset$ $L_{+}^{\alpha}(S, \mu)$ and $h_{X}(T) \subset \mathcal{M}$, respectively. On the other hand, equivalence (A.1) of the two metrics $\rho_{\mathcal{M}}$ and $\rho$ implies that $h_{f}: T \rightarrow L_{+}^{\alpha}(S, \mu)$ is Borel-measurable if and only if $h_{X}: T \rightarrow$ $\mathcal{M}$ is Borel-measurable. This, in view of Theorem 3 of [5], yields (i).

In view of Proposition 3.2 of [34], to establish (ii), we should show that any measurable $\alpha$-Fréchet process $X$ satisfies Condition S. As argued above, the map $h_{X}: t \mapsto\left[X_{t}\right]$ has a separable range in the metric space $\mathcal{L}_{0}(\Omega, \mathcal{A}, \mathrm{P})$. Hence, there exists a countable set $T_{0} \subset T$ 
such that, for all $t \in T$ and some $t_{n} \in T_{0}$, we have $X_{t_{n}} \stackrel{\mathrm{P}}{\rightarrow} X_{t}$ as $n \rightarrow \infty$. This shows that the process $X$ is separable in probability (satisfies Condition $\mathrm{S}$ ) and the proof is complete.

Proof of Theorem 4.1. Let $\left\{g_{t}\right\}_{t \in T} \subset L_{+}^{\alpha}\left(S_{I, N}, \lambda_{I, N}\right)$ be a jointly measurable minimal representation of $\left\{X_{t}\right\}_{t \in T}$ with standardized support. By Condition $S$, it suffices to focus on $\left\{f_{t}\right\}_{t \in T_{0}}$ and $\left\{g_{t}\right\}_{t \in T_{0}}$. We will show that

$$
\left\{\int_{\widetilde{S}^{N}} f_{t} \mathrm{~d} M_{\alpha}\right\}_{t \in T_{0}} \stackrel{\mathrm{D}}{=}\left\{\int_{S^{N}}^{\mathrm{e}} g_{t} \mathrm{~d} M_{\alpha}\right\}_{t \in T_{0}} \text { and }\left\{\int_{\widetilde{S}^{I}}^{\mathrm{e}} f_{t} \mathrm{~d} M_{\alpha}\right\}_{t \in T_{0}} \stackrel{\mathrm{D}}{=}\left\{\int_{S^{I}}^{\mathrm{e}} g_{t} \mathrm{~d} M_{\alpha}\right\}_{t \in T_{0}} .
$$

By Theorem 3.2 we assume without loss of generality that there exists measurable functions $\Phi: S \rightarrow S_{I, N}$ and $h: S \rightarrow \mathbb{R}_{+} \backslash\{0\}$ such that $\operatorname{supp}\left\{f_{t}: t \in T\right\}=S$ and $f_{t}(s)=h(s) g_{t} \circ$ $\Phi(s)$ for all $t \in T_{0}$ and $s \in S$. It suffices to show that

$$
\Phi\left(\tilde{S}^{N}\right)=\Phi\left(\bigcup_{\{s: \mu([s])>0\}}[s]\right)=S^{N} \quad \lambda_{I, N} \text {-a.e. }
$$

and then (A.2) will follow by a change of variables.

Observe that, since $h(s)>0$,

$$
\begin{aligned}
s \sim r & \Longleftrightarrow f_{t}(s)=q_{s, r} f_{t}(r) \quad \text { for all } t \in T_{0} \\
& \Longleftrightarrow g_{t} \circ \Phi(s)=\widetilde{q}_{s, r} g_{t} \circ \Phi(r) \text { for all } t \in T_{0} \\
& \Longleftrightarrow \Phi(s) \sim \Phi(r)
\end{aligned}
$$

for some constants $q_{s, r}>0$ and $\widetilde{q}_{s, r}>0$. Therefore, $\Phi([s])=[\Phi(s)]$ for all $s \in S$. Since $[\Phi(r)]$ is measurable (recall (4.2)), $\Phi([r])=[\Phi(r)]$ is also measurable. Now (3.3) implies that

$$
\lambda_{I, N}([\Phi(r)])=\lambda_{I, N}(\Phi([r]))=\int_{\Phi([r])} h^{\alpha} \circ \Phi^{-1}(s) \mu \circ \Phi^{-1}(\mathrm{~d} s)=\int_{[r]} h^{\alpha}(s) \mu(\mathrm{d} s),
$$

where the last equality follows from the fact that $\Phi^{-1}(\Phi([r]))=[r]$. Thus, $\mu([r])>0$ if and only if $\lambda_{I, N}([\Phi(r)])>0$ since $h(s)>0$.

We first show that $\Phi\left(\widetilde{S}^{N}\right) \subset S^{N}$ modulo $\lambda_{I, N}$. It is enough to show that, for all $r \in S$ such that $\mu([r])>0, \Phi([r])=[\Phi(r)] \subset S^{N}$ modulo $\lambda_{I, N}$. Note that (4.3) holds with $f_{t}$ replaced by $g_{t}$. It follows that the atoms of $\rho\left(g_{t}, t \in T_{0}\right)$ correspond to $\left\{[\Phi(s)], \lambda_{I, N}(\Phi(s))>0, s \in S\right\}$. In addition, observe that $\rho\left(g_{t}, t \in T_{0}\right) \sim \rho\left(g_{t}, t \in T\right)$ by Condition $\mathrm{S}$, and $\rho\left(g_{t}, t \in T\right) \sim \mathcal{B}_{S_{I, N}}$ by the minimality of $\left\{g_{t}\right\}_{t \in T}$. Therefore, for all $r \in S$ such that $\mu([r])>0$, there exists $r^{*} \in S^{N}$ such that $\lambda_{I, N}\left(\Phi([r]) \triangle\left\{r^{*}\right\}\right)=0$. This completes the proof of $\Phi\left(\widetilde{S}^{N}\right) \subset S^{N}$ modulo $\lambda_{I, N}$.

Conversely, since $\Phi$ is onto and nonsingular, for all $r^{*} \in S^{N}$ and $r \in \Phi^{-1}\left(r^{*}\right)$, we have $\Phi(\underset{\widetilde{S}}{[}])=[\Phi(r)]=\left[r^{*}\right]=\left\{r^{*}\right\}, \lambda_{I, N^{-}}$a.e. and $\mu([r])>0$. We have thus shown that $\Phi\left(\widetilde{S}^{N}\right) \supset S^{N}$ modulo $\lambda_{I, N}$.

Proof of Theorem 4.2. Part (ii) follows immediately from (4.8). To prove (i), consider another measurable representation $\left\{f_{t}^{(2)}\right\}_{t \in T} \subset L_{+}^{\alpha}\left(S_{2}, \mu_{2}\right)$ of the same process $\left\{X_{t}\right\}_{t \in T}$. We show that $\left\{f_{t}^{(2)}\right\}_{t \in T}$ also admits a co-spectral decomposition and, letting the corresponding decomposition of the process be

$$
\left\{X_{t}\right\}_{t \in T} \stackrel{\mathrm{D}}{=}\left\{\widehat{X}_{t}^{(1)} \vee \cdots \vee \widehat{X}_{t}^{(n)}\right\}_{t \in T},
$$


we have

$$
\left\{X_{t}^{(j)}\right\}_{t \in T} \stackrel{\mathrm{D}}{=}\left\{\widehat{X}_{t}^{(j)}\right\}_{t \in T}, \quad 1 \leq j \leq n .
$$

Let $\left\{f_{t}^{(1)}\right\}_{t \in T} \subset L_{+}^{\alpha}\left(S_{1}, \mu_{1}\right)$ denote the representation in assumption, which admits a cospectral decomposition with respect to $\left\{\mathcal{P}_{j}\right\}_{1 \leq j \leq n}$. Without specification, the following arguments hold for both $i=1,2$.

First, by Proposition 4.1, the process $X$ has the representation in (2.3), and, hence, it has a minimal representation with standardized support $\left\{g_{t}(s)\right\}_{t \in T} \subset L_{+}^{\alpha}\left(S_{I, N}, \lambda_{I, N}\right)$ by Theorem 3.1. This representation can also be chosen to be jointly measurable. Recall (3.2) in Theorem 3.2. By Fubini's theorem we can assume that, for all $s \in S_{i}$,

$$
f^{(i)}(s)=h_{i}(s) g . \circ \Phi_{i}(s) \quad \lambda \text {-a.e., }
$$

where $h_{i}: S_{i} \rightarrow \mathbb{R}_{+} \backslash\{0\}$ and $\Phi_{i}$ from $S_{i}$ onto $S_{I, N}$ are both measurable.

Now, suppose that $S_{1}$ has a co-spectral decomposition $S_{1}=\bigcup_{j=1}^{n} S_{1}^{(j)}$ modulo $\mu_{1}$. We show that this induces a co-spectral decomposition of $S_{I, N}$. Set

$$
S_{I, N}^{(j)}:=\Phi_{1}\left(S_{1}^{(j)}\right), \quad 1 \leq j \leq n, \quad \text { and } \quad S_{I, N}{ }^{(0)}:=S_{I, N} \backslash \bigcup_{j=1}^{n} S_{I, N}{ }^{(j)}
$$

By (A.4), $S_{I, N}{ }^{(j)} \subset\left\{s: g .(s) \in \mathcal{P}_{j}\right\}, 1 \leq j \leq n$. Note that the assumption that $S_{1}^{(j)} \cap S_{1}^{(k)} \subset$ $\left\{s \in S_{1}: f^{(1)}(s) \equiv 0\right\}$ implies that $S_{I, N}{ }^{(j)} \cap S_{I, N}{ }^{(k)} \subset\left\{s \in S_{I, N}: g .(s) \equiv 0\right\}$ for all $1 \leq j<$ $k \leq n$. Moreover, $\Phi_{1}^{-1}\left(S_{I, N}{ }^{(0)}\right) \subset S_{1} \backslash \bigcup_{j=1}^{n} S_{1}^{(j)}$, whence $\lambda_{I, N}\left(S_{I, N}{ }^{(0)}\right)=0$. We have thus shown that $\left\{S_{I, N}{ }^{(j)}\right\}_{1 \leq j \leq n}$ is a co-spectral decomposition of $\left\{g_{t}\right\}_{t \in T} \subset L_{+}^{\alpha}\left(S_{I, N}, \lambda_{I, N}\right)$ with respect to $\left\{\mathcal{P}_{j}\right\}_{1 \leq j \leq n}$.

Next, we show that, for any spectral representation $\left\{f_{t}^{(2)}\right\}_{t \in T} \subset L_{+}^{\alpha}\left(S_{2}, \mu_{2}\right)$, there exists a co-spectral decomposition of $S_{2}$ with respect to $\left\{\mathcal{P}_{j}\right\}_{1 \leq j \leq n}$. Indeed, the decomposition is induced by setting $S_{2}^{(j)}:=\Phi_{2}^{-1}\left(S_{I, N}{ }^{(j)}\right) \cap S_{2}, 1 \leq j \leq n$. We can easily verify that $\left\{S_{2}^{(j)}\right\}_{1 \leq j \leq n}$ is a co-spectral decomposition with respect to $\left\{\mathcal{P}_{j}\right\}_{1 \leq j \leq n}$.

Finally, by the construction of $\left\{S_{i}^{(j)}\right\}_{1 \leq j \leq n}, i=1,2$, above, we have

$$
\lambda_{I, N}\left(\Phi_{i}\left(S_{i}^{(j)}\right) \triangle S_{I, N}{ }^{(j)}\right)=0 \quad \text { for all } 1 \leq j \leq n .
$$

Note that (A.4) induces a max-linear isometry from $L_{+}^{\alpha}\left(S_{I, N}, \lambda_{I, N}\right)$ to $L_{+}^{\alpha}\left(S_{i}, \mu_{i}\right)$. By a change of variables, we can show that, for all $1 \leq j \leq n, m \in \mathbb{N}, a_{k} \in \mathbb{R}_{+}$, and $t_{k} \in T$,

$$
\begin{aligned}
\int_{S_{i}^{(j)}}\left(\bigvee_{k=1}^{m} \frac{f_{t_{k}}^{(i)}(s)}{a_{k}}\right)^{\alpha} \mu_{i}(\mathrm{~d} s) & =\int_{S_{i}^{(j)}}\left(\bigvee_{k=1}^{m} \frac{g_{t_{k}} \circ \Phi_{i}(s)}{a_{k}}\right)^{\alpha} h_{i}(s) \mu_{i}(\mathrm{~d} s) \\
& =\int_{S_{I, N}^{(j)}}\left(\bigvee_{k=1}^{m} \frac{g_{t_{k}}(s)}{a_{k}}\right)^{\alpha} \lambda_{I, N}(\mathrm{~d} s) .
\end{aligned}
$$

This implies (A.3).

\section{Acknowledgements}

The authors gratefully acknowledge the numerous helpful and constructive suggestions by an anonymous referee. The authors were partially supported by the NSF grant DMS-0806094 at the University of Michigan. 


\section{References}

[1] Aaronson, J. (1997). An Introduction to Infinite Ergodic Theory. American Mathematical Society, Providence, RI.

[2] Balkema, A. A. and Resnick, S. I. (1977). Max-infinite divisibility. J. Appl. Prob. 14, 309-319.

[3] Billingsley, P. (1995). Probability and Measure, 3rd edn. John Wiley, New York.

[4] Brown, B. M. and Resnick, S. I. (1977). Extreme values of independent stochastic processes. J. Appl. Prob. 14, 732-739.

[5] CoHn, D. L. (1972). Measurable choice of limit points and the existence of separable and measurable processes. Z. Wahrscheinlichkeitsth. 22, 161-165.

[6] Davis, R. A. and Resnick, S. I. (1993). Prediction of stationary max-stable processes. Ann. Appl. Prob. 3, 497-525.

[7] De HaAn, L. (1978). A characterization of multidimensional extreme-value distributions. Sankhyā A 40, 85-88.

[8] De Haan, L. (1984). A spectral representation for max-stable processes. Ann. Prob. 12, 1194-1204.

[9] De Haan, L. and Pickands, III, J. (1986). Stationary min-stable stochastic processes. Prob. Theory Relat. Fields 72, 477-492.

[10] Doob, J. L. (1953). Stochastic Processes. John Wiley, New York.

[11] Giné, E., Hahn, M. G. and Vatan, P. (1990). Max-infinitely divisible and max-stable sample continuous processes. Prob. Theory Relat. Fields 87, 139-165.

[12] Hardin, C. D., JR. (1981). Isometries on subspaces of $L^{p}$. Indiana Univ. Math. J. 30, 449-465.

[13] Hardin, C. D., JR. (1982). On the spectral representation of symmetric stable processes. J. Multivariate Anal. 12, 385-401.

[14] Kabluchko, Z. (2009). Spectral representations of sum- and max-stable processes. Extremes 12, 401-424.

[15] Kabluchko, Z. and Schlather, M. (2010). Ergodic properties of max-infinitely divisible processes. Stoch. Process. Appl. 120, 281-295.

[16] Kabluchko, Z., Schlather, M. and de HaAn, L. (2009). Stationary max-stable fields associated to negative definite functions. Ann. Prob. 37, 2042-2065.

[17] Krengel, U. (1969). Darstellungssätze für Strömungen und Halbströmungen. II. Math. Ann. 182, 1-39.

[18] Krengel, U. (1985). Ergodic Theorems. Walter de Gruyter, Berlin.

[19] OodaIRA, H. (1972). On Strassen's version of the law of the iterated logarithm for Gaussian processes. Z. Wahrscheinlichkeitsth. 21, 289-299.

[20] PipIRAs, V. (2007). Nonminimal sets, their projections and integral representations of stable processes. Stoch. Process. Appl. 117, 1285-1302.

[21] Pipiras, V. and Taqqu, M. S. (2002). The structure of self-similar stable mixed moving averages. Ann. Prob. 30, 898-932.

[22] Pipiras, V. and Taqqu, M. S. (2004). Stable stationary processes related to cyclic flows. Ann. Prob. 32, 22222260.

[23] Resnick, S. I. (1987). Extreme Values, Regular Variation, and Point Processes. Springer, New York.

[24] Resnick, S. I. ANd Roy, R. (1991). Random usc functions, max-stable processes and continuous choice. Ann. Appl. Prob. 1, 267-292.

[25] Rosiński, J. (1994). On uniqueness of the spectral representation of stable processes. J. Theoret. Prob. 7, 615634.

[26] Rosiński, J. (1995). On the structure of stationary stable processes. Ann. Prob. 23, 1163-1187.

[27] Rosiński, J. (2000). Decomposition of stationary $\alpha$-stable random fields. Ann. Prob. 28, 1797-1813.

[28] Rosiński, J. (2006). Minimal integral representations of stable processes. Prob. Math. Statist. 26, $121-142$.

[29] Rosiński, J. ANd SAMorodnitsky, G. (1996). Classes of mixing stable processes. Bernoulli 2, 365-377.

[30] Samorodnitsky, G. (2005). Null flows, positive flows and the structure of stationary symmetric stable processes. Ann. Prob. 33, 1782-1803.

[31] Samorodnitsky, G. and Taqqu, M. S. (1994). Stable Non-Gaussian Random Processes. Chapman \& Hall, New York.

[32] Sixorski, R. (1964). Boolean Algebras, 2nd edn. Academic Press, New York.

[33] Stoev, S. A. (2008). On the ergodicity and mixing of max-stable processes. Stoch. Process. Appl. 118, 16791705.

[34] Stoev, S. A. And Taqqu, M. S. (2005). Extremal stochastic integrals: a parallel between max-stable processes and $\alpha$-stable processes. Extremes 8, 237-266.

[35] Wang, Y. And Stoev, S. A. (2009). On the structure and representations of max-stable processes. Tech. Rep. 487 , Department of Statistics, University of Michigan. Available at http://arxiv.org/abs/0903.3594. 